\title{
Nonlinear ghost waves accelerate the progression of high-grade brain tumors
}

\author{
Rosa Pardo \\ Departamento de Matemática Aplicada, Facultad de Ciencias Químicas, Universidad \\ Complutense, Avda. Complutense s/n, 28040 Madrid, Spain (rpardo@mat.ucm.es) \\ Alicia Martínez-González \\ Departamento de Matemáticas,Facultad de Educación de Ciudad Real and Instituto de \\ Matemática Aplicada a la Ciencia y la Ingeniería, Universidad de Castilla-La Mancha, \\ 13071 Ciudad Real, Spain. (alicia.martinez@uclm.es) \\ Víctor M. Pérez-García \\ Departamento de Matemáticas, E. T. S. I. Industriales and Instituto de Matemática \\ Aplicada a la Ciencia y la Ingeniería, Universidad de Castilla-La Mancha, 13071 Ciudad \\ Real, Spain. victor.perezgarcia@uclm.es
}

\begin{abstract}
We study a reduced continuous model describing the evolution of high grade gliomas in response to hypoxic events through the interplay of different cellular phenotypes. We show that hypoxic events, even when sporadic and/or limited in space, may have a crucial role on the acceleration of high grade gliomas growth. Our modeling approach is based on two cellular phenotypes. One of them is more migratory and a second one is more proliferative. Transitions between both phenotypes are driven by the local oxygen values, assumed in this simple model to be uniform. Surprisingly, even very localized in time hypoxia events leading to transient migratory populations have the potential to accelerate the tumor's invasion speed up to speeds close to those of the migratory phenotype. The high invasion speed persists for times much longer than the lifetime of the hypoxic event. Moreover, the phenomenon is observed both when the migratory cells form a persistent wave of cells located on the invasion front and when they form a evanescent "ghost" wave dissapearing after a short time by decay to the more proliferative phenotype.

Our findings are obtained through numerical simulations of the model equations both in 1D and higher dimensional scenarios. We also provide a deeper mathematical analysis of some aspects of the problem such as the conditions for the existence of persistent waves of cells with a more migratory phenotype.
\end{abstract}

Keywords: High grade gliomas, Tumor hypoxia, Brain tumors, Nonlinear waves 


\section{Introduction}

Malignant gliomas are the most frequent type of primary brain tumor. Between them, the most aggressive and prevalent glioma in adults is the glioblastoma multiforme (GBM), a grade IV astrocytic tumor [54]. Mean survival after GBM diagnosis is around 14 months using the standard of care which includes surgery to resect as much tumoral tissue as possible, radiotherapy and chemotherapy (temozolamide) [36]. Despite advances in the understanding of the complex biology of these tumors, the overall prognosis has improved only slightly in the past three decades.

The main reason for treatment failure is that the periphery of the GBM typically shows tumor cells infiltrating into the normal brain, frequently even in the contralateral hemispherium. Thus, even macroscopic resection of the tumor does not eliminate many migrating cells that cause tumor recurrence $[40,6]$, typically in less than six months after surgery [22].

Pathological features of GBM are cellular pleomorphism, high cellular proliferation and diffuse infiltration, necrosis in the central regions of the tumor, microvascular hyperplasia and hypercellular areas surrounding necrotic areas around thrombosed vessels called pseudopalisades.

Due to the abnormal cell proliferation, the pre-existing vascular network is not able to appropriately feed the tumor cells. Angiogenesis emerges then in response to proangiogenic growth factors that are released by hypoxic cells in the tumor such as vascular endothelial growth factor (VEGF) [15]. The end result of VEGF signaling in tumors is the production of immature, highly permeable blood vessels with subsequent poor maintenance of the blood brain barrier and parenchymal edema [28], leading to hypoxia.

Tumor hypoxia is generally recognized as a negative clinical prognostic and predictive factor owing to its involvement in various cancer hallmarks such as resistance to cell death, angiogenesis, invasiveness, metastasis, altered metabolism and genomic instability $[45,24]$. Hypoxia plays a central role in tumor progression and resistance to therapy (chemo- and radioresistance). In GBM, it has been proven to play a key role in the biology and aggressivity of these cancers [17]. These facts have motivated considering hypoxia as a therapeutic target in cancer. Some hypoxia-regulated molecules, including hypoxia inducible factor-1 (HIF-1), carbonic anhydrase IX, glucose transporter 1, and VEGF, may be suitable targets for therapies. HIF-1 is a regulator transcriptor of cell adaptation to hypoxia efficiently translated under normoxic and hypoxic conditions, however subunit HIF-1 $\alpha$ contains an oxygen-dependent degradation domain which is rapidly degraded in normoxia.

Endothelial injury and prothrombotic factors secreted by glioma cells [13] lead to vaso-occlusive events. Following these events, necrotic regions and waves of hypoxic cells moving away from the perivascular anoxic regions $[46,7,37$, $3]$ are generated. Cells located in the perivascular areas have both oxygen and nutrients and have a high proliferative activity. However, cells exposed to hypoxia present increased migration and slower proliferation to deal with a more aggressive environment $[55,16,12]$. This phenomenon has been called the 
go-or-grow dichotomy and studied in detail in high-grade gliomas [21, 22].

Many mathematical papers have used the concept of the migration-proliferation dichotomy to explain different aspects of the behavior of tumor cell populations in vitro or in vivo $[27,49,18,43,51,25,37,38]$. Specifically, several works have considered the role of hypoxia in gliomas finding a potential beneficial effect of its reduction via either the increase of the oxygen tension in the tumour [25] or by the reduction of the occurrence of thrombotic events [37, 38].

In this work we complement previous studies and show that the effects of hypoxia may be more perverse than initially considered in previous works since transient hypoxic events may lead to accelerated progression in gliomas, even when the oxygenation is rapidly restored and persistent for very long times.

The plan of the paper is as follows. First, in Sec. 2 we present the mathematical model, some preliminary theoretical results and discuss the parameter ranges of interest. In Sec. 3 we present the results of our numerical simulations showing the acceleration of invasion by waves of cells with an hypoxic phenotype. A detailed theoretical study with some rigorous results on travelling waves of the system under study is developed in Sec. 4. In Sec. 5 we present some numerical examples of the existence of the phenomenon also in higher-dimensional scenarios. Finally in Sec. 6 we discuss the practical implications of our findings and summarize our results.

\section{The Model}

\subsection{Derivation of the Model}

Following the go-or-grow dichotomy concept we intend to describe the dynamics of the tumor in a small spatial region. We consider the tumor to be composed of two different phenotypes (compartments): a proliferative one to be denoted as $u_{n}(x, y)$ and a migratory one $u_{h}(x, y)$. We consider that the force driving phenotype changes is the local oxygen pressure. Thus, in hypoxic conditions tumor cells change to a mobile phenotype in a characteristic time $\tau_{n h}$. On the contrary, under normoxia tumor cells acquire a proliferative phenotype in a time $\tau_{h n}$.

Detailed models of these processes have been proposed in several papers (see e.g. Martínez-González et al. [37], Martinez-González et al. [38]). In this paper we use a minimal model intended to capture the essentials of a striking phenomenon: the acceleration of tumor invasion due to sporadic hypoxic events.

We assume that an initial hypoxic event around a capilar induces a complete phenotype switch of cells around it to a migratory phenotype. This is reasonable because of the fast response of HIF-1 under hypoxia, that induces phenotypic changes in times of the order of minutes [29].

Thus, we take initially our tumor density to be of the form $u_{n}(x, t=0)=$ $0, u_{h}(x, t=0)=u_{h}^{0}(x)$ and localized around a tumor vessel. Once oxygen supply is restored we assume that oxygenation is maintained above the hypoxia level for all times, may be due to the action of a therapy either normalizing 
vasculature, or avoiding thrombotic events and/or increasing oxygenation. In that scenario the dynamics is described by the equations

$$
\begin{aligned}
\frac{\partial u_{n}}{\partial t} & =D_{n} \frac{\partial^{2} u_{n}}{\partial x^{2}}+\frac{1}{\tau_{n}}\left(1-u_{n}-u_{h}\right) u_{n}+\frac{1}{\tau_{h n}} u_{h}, \\
\frac{\partial u_{h}}{\partial t} & =D_{h} \frac{\partial^{2} u_{h}}{\partial x^{2}}+\frac{1}{\tau_{h}}\left(1-u_{n}-u_{h}\right) u_{h}-\frac{1}{\tau_{h n}} u_{h} .
\end{aligned}
$$

$D_{n}, D_{h}\left(D_{h}>D_{n}\right)$ are the diffusion coefficients for the normoxic (proliferative) and hypoxic (migratory) phenotypes respectively. $\tau_{n}, \tau_{h}\left(\tau_{n}<\tau_{h}\right)$ are the doubling times for both phenotypes. This model is a pair of coupled FisherKolmogorov equations including a coupling term accounting for the decay of hypoxic cells into the normoxic phenotype with a characteristic time $\tau_{h n}$.

\subsection{Global existence and boundedness of model's solutions}

Let us first study the problem of global existence in time of non-negative solutions of Eqs. (1) with initial data $u_{n}(x, 0)=u_{n}^{0}(x), u_{h}(x, 0)=u_{h}^{0}(x)$ and $x \in \mathbb{R}, t>0$. We assume, in agreement with their biological meaning, that $D_{n}, D_{h}, \tau_{n}, \tau_{h}, \tau_{h n}>0$ are finite real parameters.

Theorem 1. For any $\left(u_{n}^{0}, u_{h}^{0}\right) \in L^{\infty} \cap H^{1}(\mathbb{R})^{2}$

(i) There exists a time $T=T\left(u_{n}^{0}, u_{h}^{0}\right)$ such that the parabolic problem (1) has a unique solution $\left(u_{n}(x, t), u_{h}(x, t)\right)$ for $0<t<T$.

(ii) Any non-negative solution $\left(u_{n}(x, t), u_{h}(x, t)\right)$ of $(1)$, is a classical solution defined globally in time, and there are constants $M_{1}, M_{2}$ such that

$$
\begin{gathered}
0 \leq u_{n}(x, t) \leq M_{1}:=\max \left\{\left\|u_{n}^{0}\right\|_{\infty}, \frac{1}{2}\left(1+\sqrt{1+4 M_{2} \tau_{n} / \tau_{h n}}\right)\right\} \\
0 \leq u_{h}(x, t) \leq M_{2}:=\max \left\{\left\|u_{h}^{0}\right\|_{\infty}, 1-\tau_{h} / \tau_{h n}\right\}
\end{gathered}
$$

Moreover, if $\tau_{h n}<\tau_{h}$, then $u_{h}(x, t) \rightarrow 0$ as $t \rightarrow \infty$.

Proof. . (i) For initial data $\left(u_{n}^{0}, u_{h}^{0}\right) \in H^{1}(\mathbb{R})^{2}$, the existence and uniqueness of mild solutions in $C\left([0, T) ; L^{2}(\mathbb{R})^{2}\right)$ holds by the variation of constants formula and standard fixed points arguments. We take $X=L^{2}(\mathbb{R})^{2} . A$ is the closure in $X$ of the differential operator $A\left(u_{n}, u_{h}\right):=\left(-D_{n} \frac{\partial^{2} u_{n}}{\partial x^{2}},-D_{h} \frac{\partial^{2} u_{h}}{\partial x^{2}}\right)$ in $C_{0}^{\infty}(\mathbb{R})^{2}$. Let us consider the fractional power spaces $X^{\alpha}:=D\left((A+I)^{\alpha}\right), \alpha \geq 0$ (see Henry [26]). In particular $X^{1 / 2}=H^{1}(\mathbb{R})^{2}$ and $X^{1}=H^{2}(\mathbb{R})^{2}$.

Let $u=\left(u_{n}, u_{h}\right), v=\left(v_{n}, v_{h}\right)$ and $f(u)=\left(f_{1}, f_{2}\right)$, be given by

$$
\begin{aligned}
& f_{1}\left(u_{n}, u_{h}\right):=\left(1-u_{n}-u_{h}\right) u_{n} / \tau_{n}+u_{h} / \tau_{h n}, \\
& f_{2}\left(u_{n}, u_{h}\right):=\left(1-u_{n}-u_{h}\right) u_{h} / \tau_{h}-u_{h} / \tau_{h n},
\end{aligned}
$$

If $1>\alpha>1 / 4$, we have

$$
\begin{aligned}
\|f(u)-f(v)\|_{L^{2}(\mathbb{R})^{2}} & \leq C\left(\|u\|_{L^{\infty}(\mathbb{R})^{2}}+\|v\|_{L^{\infty}(\mathbb{R})^{2}}\right)\|u-v\|_{L^{2}(\mathbb{R})^{2}} \\
& \leq C\left(\|u\|_{\alpha}+\|v\|_{\alpha}\right)\|u-v\|_{\alpha} \\
\|f(u)\|_{L^{2}(\mathbb{R})^{2}} & \leq C\|u\|_{L^{\infty}(\mathbb{R})^{2}}\|u\|_{L^{2}(\mathbb{R})^{2}} \leq C\|u\|_{\alpha}^{2}
\end{aligned}
$$


so the hypotheses of Henry [26, Theorem 3.3.3] are verified and local existence and uniqueness follows.

(ii) Next, we prove global existence for nonnegative solutions. To get global bounds, we use standard comparison arguments. For any non-negative solution we have that

$$
\frac{\partial u_{h}}{\partial t} \leq D_{h} \frac{\partial^{2} u_{h}}{\partial x^{2}}+\frac{1}{\tau_{h}}\left(1-u_{h}\right) u_{h}-\frac{1}{\tau_{h n}} u_{h}
$$

Therefore, since $0 \leq u_{h}^{0}(x) \leq\left\|u_{h}^{0}\right\|_{\infty}$ we obtain $0 \leq u_{h}(x, t) \leq V_{h}(t)$, where $V_{h}(t)$ is the solution of the problem

$$
\frac{d V_{h}}{d t}=\frac{1}{\tau_{h}}\left[\left(1-\frac{\tau_{h}}{\tau_{h n}}\right)-V_{h}\right] V_{h}, \quad V_{h}(0)=\left\|u_{h}^{0}\right\|_{\infty} .
$$

Let us choose $u_{h}^{0} \supsetneqq 0$. Obviously, $V_{h}(0)>0$. If $1 \leq \tau_{h} / \tau_{h n}$, then $d V_{h} / d t \leq 0$, $V_{h}(t)$ is a decreasing function on $[0, \infty)$, and moreover $V_{h}(t) \rightarrow 0$ as $t \rightarrow \infty$. Consequently $u_{h}(x, t) \rightarrow 0$ as $t \rightarrow \infty$.

Let us assume now that $1>\tau_{h} / \tau_{h n}$. If $V_{h}(0)=1-\tau_{h} / \tau_{h n}$ then $d V_{h} / d t=0$ and therefore $V_{h}(t)=1-\tau_{h} / \tau_{h n}$ on $[0, \infty)$. If $V_{h}(0)<1-\tau_{h} / \tau_{h n}$ then $d V_{h} / d t>0$ and therefore $V_{h}(t)$ is an upper-bounded increasing function on $[0, \infty)$. Thus, $V_{h}(t) \leq 1-\tau_{h} / \tau_{h n}$ on $[0, \infty)$. If $V_{h}(0)>1-\tau_{h} / \tau_{h n}$ then $d V_{h} / d t \leq 0$ and therefore $V_{h}(t)$ is a lower-bounded decreasing function on $[0, \infty)$. Thus, $V_{h}(t) \leq V_{h}(0)$ on $[0, \infty)$, and $(2 b)$ holds. Moreover, for any positive initial data, $V_{h}(t) \rightarrow$ $1-\tau_{h} / \tau_{h n}$ as $t \rightarrow \infty$.

For $u_{n}$ we get

$$
\frac{\partial u_{n}}{\partial t} \leq D_{n} \frac{\partial^{2} u_{n}}{\partial x^{2}}+\frac{1}{\tau_{n}}\left(1-u_{n}\right) u_{n}+\frac{M_{2}}{\tau_{h n}}
$$

where $M_{2}$ is given by (2b). Therefore, as in the previous case $0 \leq u_{n}(x, t) \leq$ $V_{n}(t)$, where $V_{n}(t)$ is the solution of the problem

$$
\frac{d V_{n}}{d t}=\frac{1}{\tau_{n}}\left(1-V_{n}\right) V_{n}+\frac{M_{2}}{\tau_{h n}}, \quad V_{n}(0)=\left\|u_{n}^{0}\right\|_{\infty}
$$

Let us choose $u_{n}^{0} \supsetneqq 0$, thus $V_{n}(0)>0$. If $V_{n}(0)=1 / 2+\sqrt{1 / 4+\left(M_{2} \tau_{n}\right) / \tau_{h n}}$ then $d V_{n} / d t=0$ and therefore $V_{n}(t)=V_{n}(0)$ on $[0, \infty)$. If $V_{n}(0)<1 / 2+$ $\sqrt{1 / 4+\left(M_{2} \tau_{n}\right) / \tau_{h n}}$ then $d V_{n} / d t>0$ and therefore $V_{n}(t)$ is an upper-bounded increasing function on $[0, \infty)$. Thus, $V_{n}(t) \leq 1 / 2+\sqrt{1 / 4+\left(M_{2} \tau_{n}\right) / \tau_{h n}}$ on $[0, \infty)$. If $V_{n}(0)>\frac{1}{2}+\sqrt{1 / 4+\left(M_{2} \tau_{n}\right) \tau_{h n}}$ then $d V_{n} / d t \leq 0$ and therefore $V_{n}(t)$ is a lower-bounded decreasing function on $[0, \infty)$. Thus, $V_{n}(t) \leq V_{n}(0)$. Moreover, for any positive initial data, $V_{n}(t) \rightarrow 1 / 2+\sqrt{1 / 4+\left(M_{2} \tau_{n}\right) / \tau_{h n}}$ as $t \rightarrow \infty$. Hence, (2a) holds, which completes the proof.

Proposition 2. Assume that $\tau_{h n}>\tau_{n}$. The box

$$
\Sigma:=\left\{\left(u_{n}, u_{h}\right): 0 \leq u_{n}, u_{h} \leq 1\right\}
$$

is an invariant box for (1), i.e. for any initial data $\left(u_{n}^{0}, u_{h}^{0}\right) \in \Sigma$, the solution of $(1)\left(u_{n}(x, t), u_{h}(x, t)\right) \in \Sigma$ for any $t \geq 0$. 
Proof. Taking $f$ as given by Eqs. (3), assuming that $\left(u_{n}, u_{h}\right) \in \Sigma$, and that $\tau_{h n}>\tau_{n}$, then

$$
\begin{array}{cl}
f_{1}\left(0, u_{h}\right):=u_{h} / \tau_{h n} \geq 0, & f_{1}\left(1, u_{h}\right):=\left(1 / \tau_{h n}-1 / \tau_{n}\right) u_{h} \leq 0 \\
f_{2}\left(u_{n}, 0\right):=0, & f_{2}\left(u_{n}, 1\right):=-u_{n} / \tau_{h}-1 / \tau_{h n} \leq 0 .
\end{array}
$$

Therefore, $\Sigma$ is an invariant box, see Smoller [48, Section 14.B, p. 198].

\subsection{Parameter estimation}

Brain tissue has a complex structure with spatial inhomogeneities in the parameter values (e.g. different propagation speeds in white and gray matter) and anisotropies (e.g. on the diffusion tensor with preferential propagation directions along white matter tracts). In order to focus our attention on the essential features of the phenomena to be studied, we have chosen to study first the model in one spatial dimension and in isotropic media. In Sec. 5 we will complement our analysis with some higher-dimensional examples.

In Eqs. (1), the densities $u_{n}, u_{h}$ are measured in units of the maximal tissue density, typically around $10^{3} \mathrm{cell} / \mathrm{cm}$. The normoxic cell doubling time will be taken to be $\tau_{n} \sim 24 \mathrm{~h}$ in agreement with typical cell doubling times in vitro (see e.g. Ke et al. [30]) and the hypoxic one $\tau_{h} \sim 48 \mathrm{~h}$. The diffusion coefficients for normoxic and hypoxic cells are around $D_{n}=6.6 \cdot 10^{-12} \mathrm{~cm}^{2} / \mathrm{s}$ and $D_{h}$ approximately an order of magnitude larger [37]. The parameter $\tau_{h n}$, corresponding to the phenotype switch time is harder to estimate. It measures the time of recovery of the proliferative phenotype in normoxic conditions. Normal cells have the capability of restoring their normal behavior when oxygenation is restored. However, cancer cells may become more aggressive after several cycles of hypoxia and reoxigenation $[4,3]$. The process may become at some moment irreversible leading to the so-called Warburg phenotype [33, 39]. Different works point out to a normalization of the response to hypoxia between $48 \mathrm{~h}$ and 72 $\mathrm{h}$ [52]. In vivo analysis of HIF- $1 \alpha$ stabilization in well oxygenated tumor areas [55] provides support for long normalization times $\tau_{h n}$.

To solve Eqs. (1) numerically we have used a standard finite difference method of second order in time and space with zero boundary conditions on the boundaries of the integration domain. We have used large integration domains and cross-checked our results for different domain sizes to avoid boundary effects.

\section{Numerical results}

\subsection{Hypoxic events lead to fast glioma progression}

An example of the phenomenon to be described here is presented in Figs. 1 and 2. There, an initial distribution of hypoxic tumor cells at $t=0$, e.g. originated after a transient vaso-occlusive event, is placed in a well oxygenated environment. One might expect naively that after a transient of about a few times $\tau_{h n}$, hypoxic cells would dissapear and then the front speed would tend 

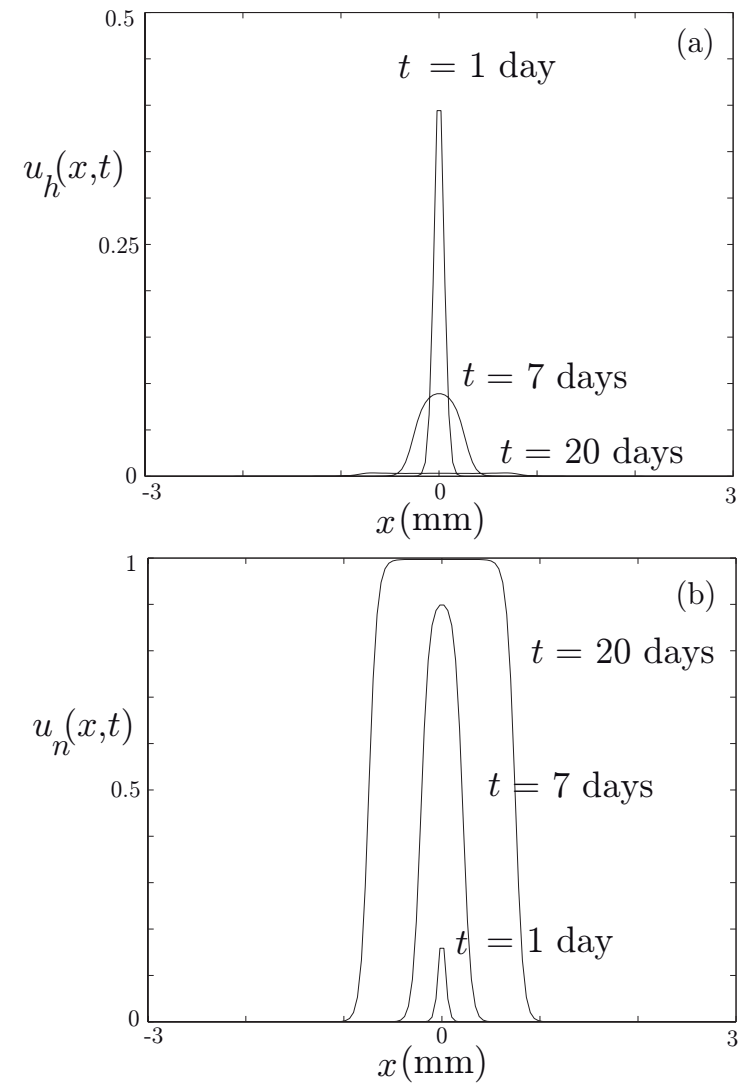

Figure 1: Snapshots of the evolution of the (a) hypoxic $u_{h}(x, t)$ and (b) normoxic $u_{n}(x, t)$ densities solving Eq. (1) for parameter values $D_{n}=6.6 \times 10^{-12} \mathrm{~cm}^{2} \mathrm{~s}^{-1}, D_{h}=1.32 \times 10^{-10}$ $\mathrm{cm}^{2} \mathrm{~s}^{-1}, \tau_{n}=24 \mathrm{~h}, \tau_{h}=48 \mathrm{~h}, \tau_{h n}=96 \mathrm{~h}$ and initial data $u_{n}(x, 0)=0, u_{h}(x, 0)=$ $\left(1-800 x^{2}\right)_{+}, x$ being measured in mm. Shownare the results for $t=1,7$ and 20 days showing the decay of the hypoxic cell density and the formation of a travelling wave of normoxic cells. 
asymptotically to that of the normoxic phenotype, given by the minimal FK speed

$$
c_{n}^{*}=2 \sqrt{D_{n} / \tau_{n}}
$$

Although the hypoxic cell density decays and a front of normoxic cells is generated (see Fig. 1), the normoxic front propagation speed is not the minimal FK speed. In Fig. 2 we show pseudocolor spatio-temporal plots of $u_{n}(x, t)$ (Fig. 2(a)) and the front speed (Fig. 2(b)). The internal and external white lines show (respectively) the predicted evolution of purely normoxic and hypoxic initial data under no phenotype changes (i.e., the limit $\tau_{h n} \rightarrow \infty$ ).

From the results shown in Fig. 2 it is clear that the speed of the propagating front of normoxic cells is not $c_{n}^{*}$ but given by a substantially larger value even when the hypoxic cell population has become extinct (cf. Fig. 2(c)), i.e. the decaying "ghost" waves have a substantial effect on the final invasion speed of the tumor. The simulation runs for long times to show that, although the hypoxic cell amplitude decays in a few days, its effect on the normoxic front propagation speed persists and provides a sustained front acceleration that is still present (though minimal) after one month of the initial $\left(t=0^{-}\right)$hypoxic event.

\section{2. "Ghost" waves of hypoxic cells drive the evolution of the normoxic compo- nent}

To confirm that the acceleration of the normoxic phenotype front is due to the presence of the hypoxic component we have performed a second set of numerical experiments. In these simulations we have studied the effect of the suppression of the hypoxic component at a given time on the final propagation speed. Thus we have solved Eqs. (1) but setting artificially $u_{h}\left(x, t_{\text {sup }}\right)=0$ for different values of $t_{\text {sup }}$.

The results are summarized in Fig. 3. Note that for the simulations of Fig. 3 we have chosen a substantially smaller $\tau_{h n}$ leading to a faster decay of the amplitude of the hypoxic component. It is remarkable that when the hypoxic wave amplitude is very small, it continues accelerating the front of normoxic phenotype cells. This behavior is somehow counterintuitive implying that the final normoxic wave speed more than doubles the expected speed $c_{n}$.

To understand this phenomenon intuitively, let us note that initially the hypoxic wave propagates with speed

$$
c_{h}^{*}=2 \sqrt{D_{h} / \tau_{h}}
$$

with $c_{h}^{*}>c_{n}^{*}$. At the same time, the hypoxic wave amplitude decreases while diffusing through the medium. This provides a seeding of hypoxic cells that change their phenotype to normoxic thus providing an extra source for normoxic cells. It is interesting that the phenomenon is mediated by a small amplitude wave of hypoxic cells. This leads to a substantially faster growth of the normoxic component when the wave reaches a specific location. The outcome of this dynamical phenomenon is that the effects of an initial hypoxic event may influence the tumor growth speed for long times. 

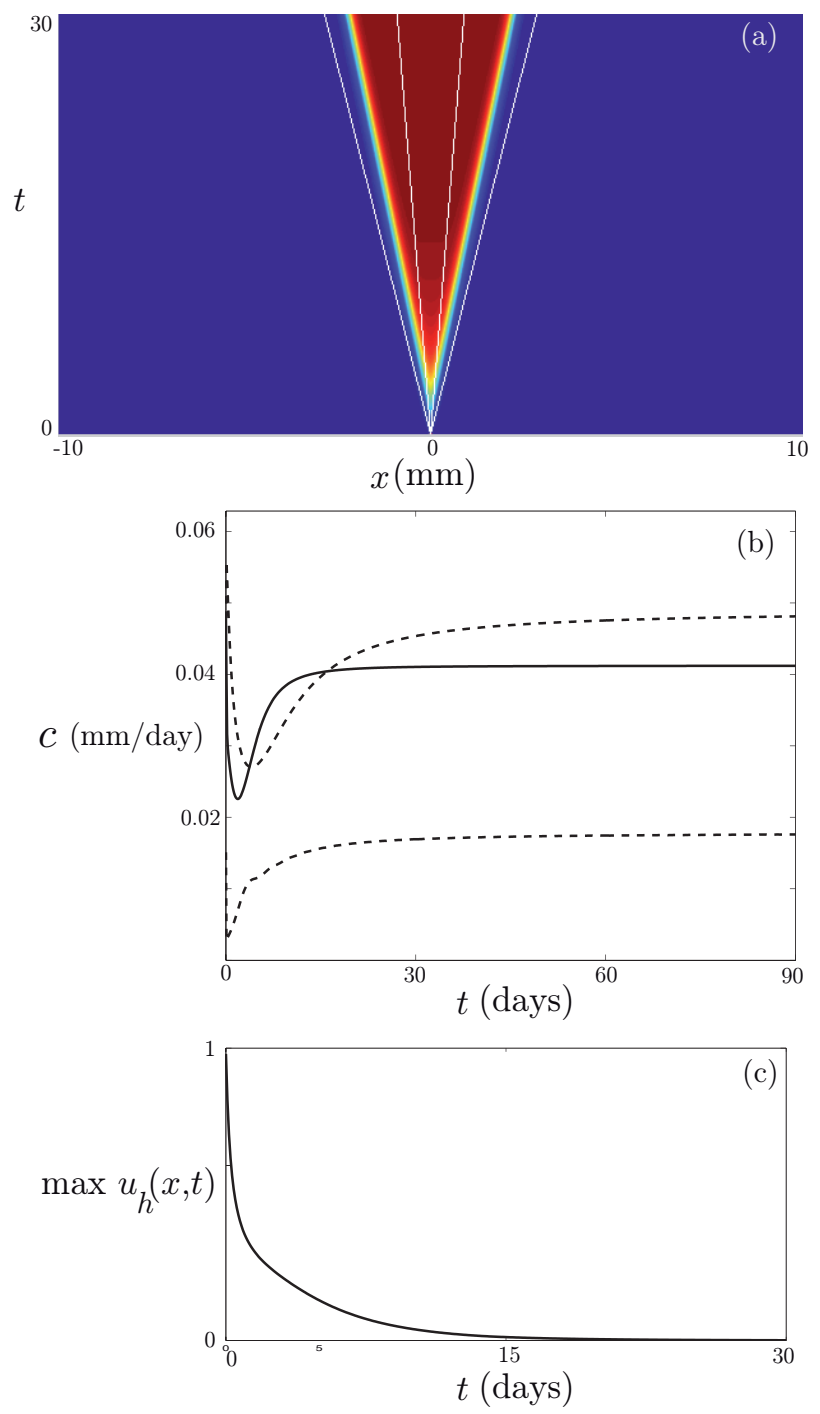

Figure 2: Evolution of the normoxic $u_{n}(x, t)$ and hypoxic $u_{h}(x, t)$ cell densities solving Eq. (1) for parameter values $D_{n}=6.6 \times 10^{-12} \mathrm{~cm}^{2} \mathrm{~s}^{-1}, D_{h}=1.32 \times 10^{-10} \mathrm{~cm}^{2} \mathrm{~s}^{-1}, \tau_{n}=24 \mathrm{~h}, \tau_{h}=$ $48 \mathrm{~h}, \tau_{h n}=96 \mathrm{~h}$ and localized initial data of the form $u_{n}(x, 0)=0, u_{h}(x, 0)=\left(1-800 x^{2}\right)_{+}$, $x$ being measured in mm. (a) Pseudocolor plots of the amplitude of the normoxic cell density $u_{n}(x, t)$. The white lines are calculated using the propagation speed of the hypoxic and normoxic fronts in the framework of the FK equation (b) Evolution of the normoxic wavefront speed calculated as $v(t)=\frac{d}{d t}\left[2\left(\int|x-X(t)| u_{n}(x, t) d x\right) /\left(\int u_{n}(x, t) d x\right)\right]$, where $X(t)=\int x u_{n}(x, t) d x$. Shownare for comparison the speeds (calculated using the same formula) for purely hypoxic (upper dashed line) and purely normoxic cells (dashed lower line) without any type of transitions allowed between them. The long time scales shown guarantee that the front reaches an asymptotic speed, found to be substantially larger than that of the normoxic front. (c) Decay of the amplitude of the hypoxic component $\max _{x} u_{h}(x, t)$, as a function of time. 


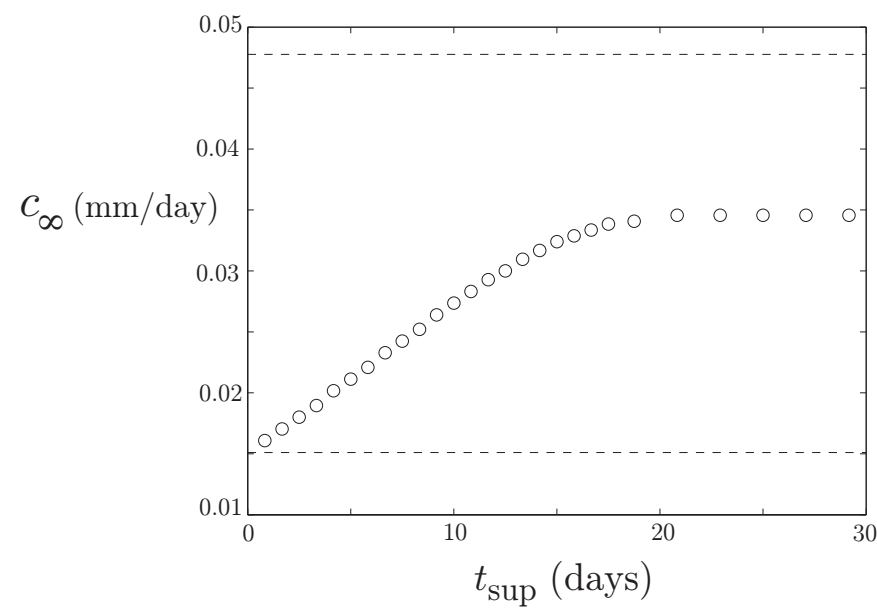

Figure 3: (a) Effect on the final asymptotic speed at $t=30$ days of the suppression of the hypoxic component for different times $t_{\text {sup. }}$. The horizontal dashed lines denote the normoxic $c_{n}$ (lower line) and hypoxic $c_{h}$ (upper line) phenotype speeds respectively. (b) Decay of the amplitude of the hypoxic cell subpopulation. Parameter values are $D_{n}=6.6 \times 10^{-12} \mathrm{~cm}^{2} \mathrm{~s}^{-1}$, $D_{h}=1.32 \times 10^{-10} \mathrm{~cm}^{2} \mathrm{~s}^{-1}, \tau_{n}=24 \mathrm{~h}, \tau_{h}=48 \mathrm{~h}, \tau_{h n}=48 \mathrm{~h}$ and initial data as in Fig. 2.

One may wonder if the outcome of our simulations may be due to the fact that the hypoxic initial distribution is not surrounded by normoxic tumor cells as it may happen in real tissue. To rule out this possibility we have run simulations with initial data localized around a vessel but surrounded by normoxic cells. An example of our results is shown in Fig. 4 ruling out the influence this choice on the asymptotic speed.

It is important to emphasize that the phenomenon described in this section occurs in broad parameter regions including the biologically relevant ranges of parameters. As an example, in Fig. 5 we plot the asymptotic speed as a function of the normoxic doubling time ranging from the typical in vitro values of $\tau_{n}=24$ $\mathrm{h}$ to larger values closer to in-vivo doubling time estimates [53, 31]

\subsection{Propagation regimes as a function of $\tau_{h n}$}

The parameter $\tau_{h n}$ describes complicated biological processes since it corresponds to the phenotype change of hypoxic cells under oxic conditions. As described in Sec. 2, this parameter can range from about an hour in normal cells to many days in transformed cells. One would expect that very fast decaying hypoxic phenotypes would lead to a smaller acceleration of the normoxic wave of invasion. In the opposite limit it is to expected that the asymptotic front velocity $c_{\infty}$ satisfies $\lim _{\tau_{h n} \rightarrow \infty} c_{\infty}\left(\tau_{h n}\right)=c_{h}^{*}$.

In Fig. 6 we explore the behavior of the asymptotic velocity of the normoxic wave one month after the hypoxic event for different values of the switch parameter $\tau_{h n}$. It can be seen how larger recovery times lead to asymptotic speeds closer to $c_{h}^{*}$. 


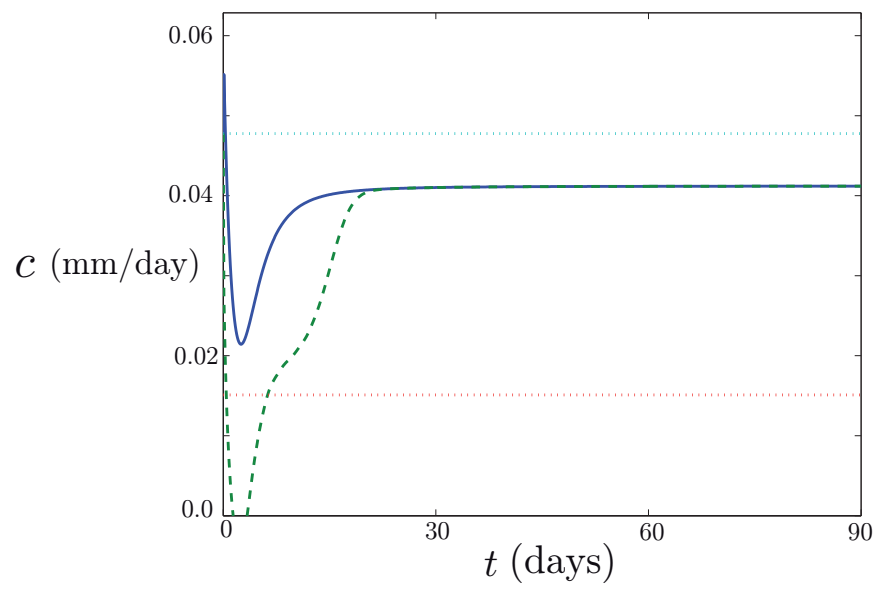

Figure 4: Tumor growth speeds for parameter values $D_{n}=6.6 \times 10^{-12} \mathrm{~cm}^{2} \mathrm{~s}^{-1}, D_{h}=$ $1.32 \times 10^{-10} \mathrm{~cm}^{2} \mathrm{~s}^{-1}, \tau_{n}=24 \mathrm{~h}, \tau_{h}=48 \mathrm{~h}, \tau_{h n}=96 \mathrm{~h}$ and different initial data. The solid line corresponds to the same situation as in Fig. 2, i.e. initial data of the form $u_{n}(x, 0)=0, u_{h}(x, 0)=\left(1-800 x^{2}\right)_{+}, x$ being measured in mm. The dashed lines correspond to the evolution of initial data of the form $u_{n}(x, 0)=0.72\left[1-200(x-0.2)^{2}\right]_{+}+$ $0.72\left[1-200(x+0.2)^{2}\right]_{+}, u_{h}(x, 0)=\left(1-800 x^{2}\right)$ corresponding to broad normoxic tumor cell densities around vessels adjacent to the one failing and generating the localized hypoxic density $u_{h}$. Shownare for comparison the asymptotic speeds of purely hypoxic (upper dotted line) and purely normoxic cells (lower dotted line) calculated using the propagation speed of the hypoxic and normoxic fronts in the framework of the standard scalar FK equation.

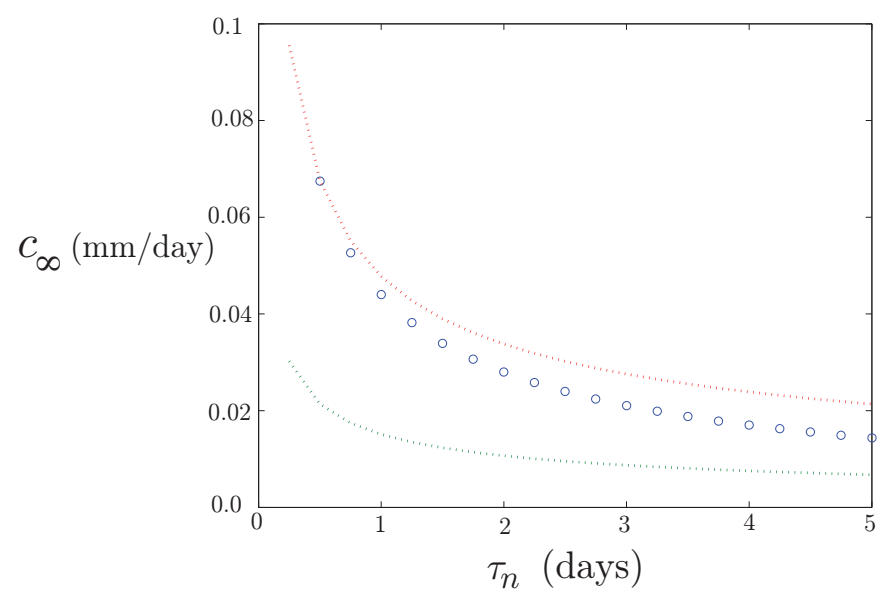

Figure 5: Asymptotic tumor growth speed as a function of the normoxic proliferation time $\tau_{n}$. The hypoxic proliferation time is chosen to be twice the value of $\tau_{n}$, i.e. $\tau_{h}=2 \tau_{n}$. The dashed lines denote the normoxic $c_{n}^{*}$ (lower dashed line) and hypoxic $c_{h}^{*}$ (upper line) phenotype asymptotic speeds respectively. Parameter values are $D_{n}=6.6 \times 10^{-12} \mathrm{~cm}^{2} \mathrm{~s}^{-1}$, $D_{h}=1.32 \times 10^{-10} \mathrm{~cm}^{2} \mathrm{~s}^{-1}, \tau_{h n}=7$ days and initial data as in Fig. 2 . 


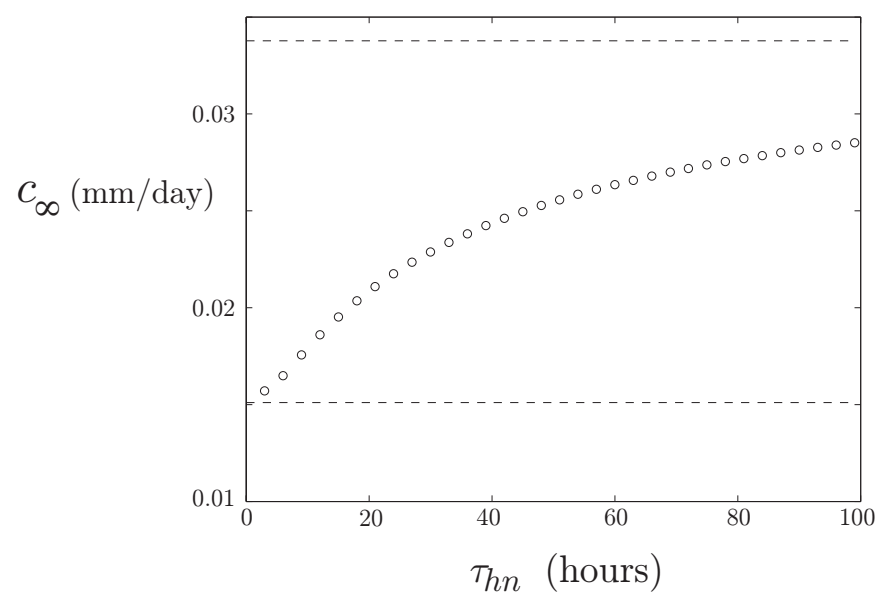

Figure 6: Asymptotic speed $c_{\infty}$ of the solutions of Eqs. (1) after one month as a function of the switch time $\tau_{h n}$. Parameter values $\tau_{n}=24 \mathrm{~h}, \tau_{h}=48 \mathrm{~h}, D_{n}=6.6 \times 10^{-12} \mathrm{~cm}^{2} \mathrm{~s}^{-1}, D_{h}=$ $6.6 \times 10^{-11} \mathrm{~cm}^{2} \mathrm{~s}^{-1}$ and initial data as in Fig. 2. The dashed lines represent the minimal asymptotic speeds of hypoxic $c_{h}^{*}$ (upper line) and normoxic $c_{n}^{*}$ invasion waves (lower line).

\subsection{Localized finite-amplitude wavepackets of hypoxic cells}

It is very interesting that despite the instability of the hypoxic cells, there are parameter regimes in which the hypoxic cells do not form an evanescent wave. Instead, a finite amplitude wavepacket of cells with the hypoxic phenotype persists leading the advance of the tumor.

Typical results are shown in Fig. 7. As it can be seen in the pseudocolor plot of Fig. 7(a) and in Fig. 7(c), the hypoxic cell density does not vanish with time since their fast proliferation rates makes the zero solution unstable when the hypoxic wave hits the normal tissue areas. However, the hypoxic solution is unstable and decays into the normoxic one leading to a bright soliton of hypoxic cells (cf. Fig. $7(\mathrm{c})$ ) coupled to the front corresponding to the normoxic phenotype (Fig. 7(b)). The speed of the resulting wave of hypoxic cells becomes asymptotically close to $c_{h}^{*}$. From the mathematical point of view these solutions correspond to stable homoclinic orbits of the system connecting with the equilibrium point $u_{h}=0$.

These families of solutions may correspond to the behavior of very aggressive phenotypes driven by hypoxia with both enhanced mobility and proliferation. In general, it is found in the context of different families of models that more aggressive phenotypes tend to be the drivers of invasion [44, 1]. What it is interesting in the result described here is the fact that even unstable phenotypes may support a robust non-vanishing wave. 

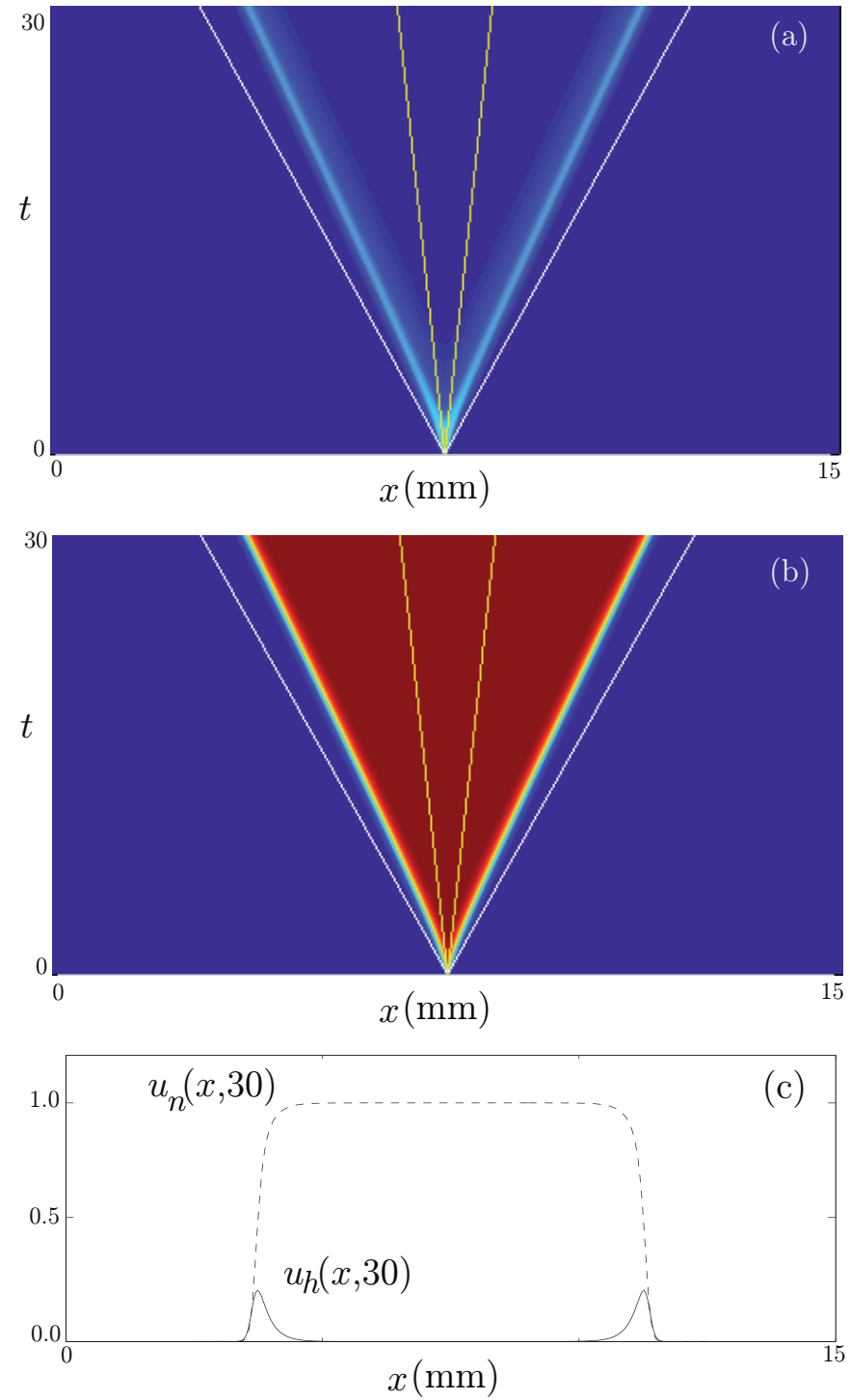

Figure 7: Evolution of the normoxic $u_{n}(x, t)$ and hypoxic $u_{h}(x, t)$ cell densities solving Eq. (1) for parameter values $D_{n}=6.6 \times 10^{-12} \mathrm{~cm}^{2} \mathrm{~s}^{-1}, D_{h}=1.32 \times 10^{-10} \mathrm{~cm}^{2} \mathrm{~s}^{-1}, \tau_{n}=24 \mathrm{~h}, \tau_{h}=$ $18 \mathrm{~h}, \tau_{h n}=72 \mathrm{~h}$ and localized initial data of the form $u_{n}(x, 0)=0, u_{h}(x, 0)=\left(1-800 x^{2}\right)_{+}$, $x$ being measured in mm. (a,b) Pseudocolor plots of the amplitude of the hypoxic (a) and normoxic (b) cell densities $u_{h}(x, t)$ and $u_{n}(x, t)$ respectively. The lines shown are calculated using the propagation speed of the hypoxic and normoxic fronts in the framework of the FK model (c) Final profiles of the normoxic and hypoxic cell densities after $t=30$ days showing the coupled front and bright soliton. A small amplitude pulse of hypoxic cells leads the propagation of the tumor despite the instability of the zero solution. 


\section{Theoretical results}

In the previous sections we have described several phenomena involving the existence of both decaying and localized travelling waves of hypoxic cells. In this section we wish to complement our numerical results with some theory on the existence of localized finite-amplitude waves of the system studied.

Our main results in this section are Theorem 11 and Corollary 13. Theorem 11 provides necessary conditions for having bifurcation from the solution $\left(v_{K}, 0\right)$, where $v_{K}$ denotes the classical KPP solution to the Fisher problem, cf. [19, 32]. Corollary 13 states that the 'smallest component' of the hypoxic wave propagates with speed

$$
c_{h n}^{*}:=2 \sqrt{D_{h}\left(\frac{1}{\tau_{h}}-\frac{1}{\tau_{h n}}\right)} .
$$

Let $\left(u_{n}, u_{h}\right)$ be a solution of (1). Under some assumptions on the parameters to be made more precise later, Eqs. (1) admit travelling wave solutions of the form $u_{n}(x, t)=v_{n}(x-c t), u_{h}(x, t)=v_{h}(x-c t)$, for some values of $c>0$.

Let $\xi:=x-c t$. The functions $\left(v_{n}, v_{h}\right)=\left(v_{n}(\xi), v_{h}(\xi)\right)$ satisfy the equations

$$
\begin{aligned}
& -D_{n} v_{n}^{\prime \prime}-c v_{n}^{\prime}=\left(1-v_{n}-v_{h}\right) v_{n} / \tau_{n}+v_{h} / \tau_{h n}, \\
& -D_{h} v_{h}^{\prime \prime}-c v_{h}^{\prime}=\left(1-v_{n}-v_{h}\right) v_{h} / \tau_{h}-v_{h} / \tau_{h n}, \quad \text { for } \quad \xi \in \mathbb{R} .
\end{aligned}
$$

where the prime denotes differentiation with respect to $\xi$.

We will look for solutions $\left(v_{n}^{*}, v_{h}^{*}\right)$ such that

$$
\begin{aligned}
& \left(v_{n}^{*},\left(v_{n}^{*}\right)^{\prime}, v_{h}^{*},\left(v_{h}^{*}\right)^{\prime}\right) \rightarrow(0,0,0,0) \quad \text { as } \xi \rightarrow+\infty \\
& \left(v_{n}^{*},\left(v_{n}^{*}\right)^{\prime}, v_{h}^{*},\left(v_{h}^{*}\right)^{\prime}\right) \rightarrow(1,0,0,0) \quad \text { as } \xi \rightarrow-\infty,
\end{aligned}
$$

with $v_{n}^{*}, v_{h}^{*}>0$.

Obviously, choosing $\left(v_{n}, v_{h}\right)=\left(v_{K}, 0\right)$ where $v_{K}$ denotes the classical KPP solution to the Fisher problem, see $[19,32]$, we get a solution of (12). It is well known that whenever $c>c_{n}^{*}\left(\right.$ or $\left.c<-c_{n}^{*}\right)$, see (10a) for a definition of $c_{n}^{*}$, there exists a travelling wave $v_{K}$, solving

$$
-D_{n} v_{K}^{\prime \prime}-c v_{K}^{\prime}=\left(1-v_{K}\right) v_{K} / \tau_{n}, \quad \text { for } \quad \xi \in \mathbb{R}
$$

and satisfying $0<v_{K}<1$,

$$
\left\{\begin{array}{l}
v_{K}(\xi) \rightarrow 0 \text { as } \xi \rightarrow+\infty \\
v_{K}(\xi) \rightarrow 1 \text { as } \xi \rightarrow-\infty \\
v_{K}^{\prime}(\xi) \rightarrow 0 \text { as }|\xi| \rightarrow \infty
\end{array}\right.
$$

This solution corresponds to the heteroclinic orbit of the associated first order ordinary differential system, connecting the critical point $(1,0)$ with $(0,0)$. 
Here we consider solutions with $v_{h}>0$ and analyze the associated first order ordinary differential system, see (A.1), and its critical points. We refer to the Appendix Appendix A for an study of the ODE, its equilibria and their corresponding stability. See Theorems 17 , and 19 where we prove that $(0,0,0,0)$ is an stable equilibrium whenever $\tau_{h n}>\tau_{h}$, and $(1,0,0,0)$ is a saddle point with a 2-dimensional unstable manifold $E^{u}$ (and also a 2-dimensional stable manifold $\left.E^{s}\right)$.

Theorem 3. Let us denote by $V=\left(V_{1}, V_{2}, V_{3}, V_{4}\right)=\left(v_{n}, v_{n}^{\prime}, v_{h}, v_{h}^{\prime}\right), V=V(\xi)$, a solution of the ordinary differential equation (A.1). Assume that $\tau_{h n}>\tau_{h}$ and $c>\max \left\{c_{n}^{*}, c_{h n}^{*}\right\}$, where

$$
c_{h n}^{*}:=2 \sqrt{D_{h}\left(1 / \tau_{h}-1 / \tau_{h n}\right)} .
$$

Let $E^{u}$ be the 2-dimensional unstable manifold of the equilibrium $(1,0,0,0)$ characterized in Theorem 19. Then, for any $V_{0} \in E^{u}$ there is a trajectory $V=V\left(\xi ; V_{0}\right) \rightarrow(0,0,0,0)$ as $\xi \rightarrow+\infty$, and $V=V\left(\xi ; V_{0}\right) \rightarrow(1,0,0,0)$ as $\xi \rightarrow-\infty$.

Using this result, to be proven later in this paper, we can move to the following theorem, that proves the existence of a 2-dimensional manifold of initial data of heteroclinic trajectories of the associated ODE (A.1), connecting the critical points $(1,0,0,0)$ with $(0,0,0,0)$. The intersection of the 2 -dimensional manifold of initial data of heteroclinic trajectories with $[0,1] \times \mathbb{R} \times[0,1] \times \mathbb{R}$ is a set of initial data for travelling waves. The idea of the proof is to choose a point on the unstable 2-dimensional manifold $E^{u}$ of the equilibrium $(1,0,0,0)$ and prove that, for some range of speeds, the trajectory falls down into the basin of attraction of $(0,0,0,0)$.

Theorem 4. Assume that $\tau_{h n}>\tau_{h}$ and $c>\max \left\{c_{n}^{*}, c_{h n}^{*}\right\}$, where $c_{n}^{*}, c_{h n}^{*}$ are defined in (10a), (16) respectively. Let $E^{u}$ be the real 2-dimensional unstable manifold of the equilibrium (1,0,0,0) characterized in Theorem 19.

Then, for any $V_{0} \in E^{u} \cap[0,1] \times \mathbb{R} \times[0,1] \times \mathbb{R} \neq \emptyset$, there exists a travelling wave $\left(v_{n}^{*}, v_{h}^{*}\right)$ solving $(12)$, satisfying $(13)$, and such that

$$
\left.\left(v_{n}^{*},\left(v_{n}^{*}\right)^{\prime}, v_{h}^{*},\left(v_{h}^{*}\right)^{\prime}\right)\right|_{\xi=\xi_{0}}=V_{0} \quad \text { for some } \xi_{0} \in \mathbb{R} .
$$

Proof. Let $\left(v_{n}, v_{h}\right)$ be a solution of $(12)$ and $V(\xi)=\left(V_{1}, V_{2}, V_{3}, V_{4}\right)=\left(v_{n}, v_{n}^{\prime}, v_{h}, v_{h}^{\prime}\right)$, satisfying Eq. (A.1).

From Theorem 3, there is a real 2-dimensional manifold of the equilibrium $E^{u}$ such that, for any initial data $V_{0} \in E^{u}$, the trajectory $V=V\left(\xi ; V_{0}\right) \rightarrow(1,0,0,0)$ as $\xi \rightarrow-\infty$, and $V=V\left(\xi ; V_{0}\right) \rightarrow(0,0,0,0)$ as $\xi \rightarrow \infty$.

Obviously $\left.\left(v_{K}, v_{K}^{\prime}, 0,0\right)\right|_{\xi_{1}} \in E^{u} \cap[0,1] \times \mathbb{R} \times[0,1] \times \mathbb{R}$ and $v_{K}\left(\xi_{1}\right)<1$. Let us consider $V_{0} \in E^{u} \cap[0,1] \times \mathbb{R} \times[0,1] \times \mathbb{R} \neq \emptyset$, then $\left(v_{n}, v_{h}\right)$ satisfies the required conditions.

Next, we plan to analyze the bifurcation from $\left(v_{n}, v_{h}\right)=\left(v_{K}, 0\right)$ to get conditions for having $v_{n}, v_{h}>0$. Let us denote by $z=\left(z_{n}, z_{h}\right):=\left(v_{n}-v_{K}, v_{h}\right)$, 
then $z$ satisfies the equations

$$
\begin{aligned}
& L_{1} z_{n}=\left(2-z_{n}-z_{h}\right) z_{n} / \tau_{n}+\left(1 / \tau_{h n}-1 / \tau_{n} v_{K}\right) z_{h}, \\
& L_{2} z_{h}=\left(2-z_{n}-z_{h}\right) z_{h} / \tau_{h}-z_{h} / \tau_{h n}, \quad \text { for } \quad \xi \in \mathbb{R},
\end{aligned}
$$

where,

$$
\begin{aligned}
& L_{1}:=-D_{n} \frac{d^{2}}{d \xi^{2}}-c \frac{d}{d \xi}+\frac{2}{\tau_{n}} v_{K}+\frac{1}{\tau_{n}}, \\
& L_{2}:=-D_{h} \frac{d^{2}}{d \xi^{2}}-c \frac{d}{d \xi}+\frac{1}{\tau_{h}} v_{K}+\frac{1}{\tau_{h}},
\end{aligned}
$$

in order to avoid that $0 \in \sigma\left(L_{1}\right)$ or $0 \in \sigma\left(L_{2}\right)$, we add $z_{n} / \tau_{n}$ and $z_{h} / \tau_{h}$ respectively to both sides, consequently $\sigma\left(L_{1}\right) \subset\left[1 / \tau_{n}, \infty\right)$ and $\in \sigma\left(L_{2}\right) \subset\left[1 / \tau_{h}, \infty\right)$ respectively, see [2].

We look for solutions $\left(z_{n}, z_{h}\right)$ such that $\left(z_{n},\left(z_{n}\right)^{\prime}, z_{h},\left(z_{h}\right)^{\prime}\right) \rightarrow(0,0,0,0)$ with $z_{h}>0$, as $|\xi| \rightarrow+\infty$. Let $\left(z_{n}, z_{h}\right)$ be a solution of (17) and let us define $Z=\left(Z_{1}, Z_{2}, Z_{3}, Z_{4}\right)=\left(z_{n}, z_{n}^{\prime}, z_{h}, z_{h}^{\prime}\right)$. Then $Z=Z(\xi)$, satisfies the equations

$$
\frac{d Z}{d \xi}=A Z+B(\xi) Z+f(\xi, Z)
$$

where

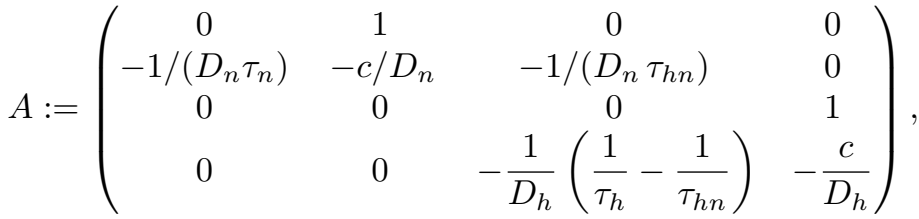

$$
\begin{aligned}
& B(\xi):=\left(\begin{array}{cccc}
0 & 0 & 0 & 0 \\
2 \frac{v_{K}}{D_{n} \tau_{n}} & 0 & \frac{v_{K}}{D_{n} \tau_{n}} & 0 \\
0 & 0 & 0 & 0 \\
0 & 0 & \frac{v_{K}}{D_{h} \tau_{h}} & 0
\end{array}\right), f(\xi, Z):=\left(\begin{array}{c}
0 \\
\frac{1}{D_{n} \tau_{n}}\left(Z_{1}+Z_{3}\right) Z_{1} \\
0 \\
\frac{1}{D_{h} \tau_{h}}\left(Z_{1}+Z_{3}\right) Z_{3}
\end{array}\right)
\end{aligned}
$$

Using (15), we get $\lim _{\xi \rightarrow+\infty} B(\xi)=: B(+\infty)=\left(\begin{array}{cccc}0 & 0 & 0 & 0 \\ 0 & 0 & 0 & 0 \\ 0 & 0 & 0 & 0 \\ 0 & 0 & 0 & 0\end{array}\right)$, and

$$
\lim _{\xi \rightarrow-\infty} B(\xi)=: B(-\infty)=\left(\begin{array}{cccc}
0 & 0 & 0 & 0 \\
\frac{2}{D_{n} \tau_{n}} & 0 & \frac{1}{D_{n} \tau_{n}} & 0 \\
0 & 0 & 0 & 0 \\
0 & 0 & \frac{1}{D_{h} \tau_{h}} & 0
\end{array}\right) .
$$

The following proposition gives information on the asymptotic behavior of a solution of (19a), by using classical perturbation theory [14, Chapter 13]. Specifically, we prove that the minimal speed of the purely hypoxic wave is given by $c_{h n}^{*}$. defined in Eq. (16). 
Proposition 5. Let $Z=\left(Z_{1}, Z_{2}, Z_{3}, Z_{4}\right), \quad Z=Z(\xi)$, be a solution of (19a). Assume that $\tau_{h n}>\tau_{h}$ and $c>\max \left\{c_{n}^{*}, c_{h n}^{*}\right\}$, where $c_{n}^{*}, c_{h n}^{*}$ are defined in (10a), (16) respectively. Then

(i) $\sigma(A):=\left\{\lambda_{1 \pm}, \lambda_{2 \pm}\right\} \subset \mathbb{R}$, where $\lambda_{1 \pm}, \lambda_{2 \pm}$ are defined in (21), $\mu_{+\infty}:=$ $\max \sigma(A)<0$, and the trivial solution is asymptotically stable. Moreover, if $\left|Z(0)=\left(z_{n}(0), z_{n}^{\prime}(0), z_{h}(0), z_{h}^{\prime}(0)\right)\right|$ is sufficiently small, then

$$
\limsup _{\xi \rightarrow+\infty} \frac{\log |Z(\xi)|}{\xi} \leq \mu_{+\infty}<0,
$$

where $Z(\xi)=\left(z_{n}(\xi), z_{n}^{\prime}(\xi), z_{h}(\xi), z_{h}^{\prime}(\xi)\right)$.

(ii) $\sigma(A+B(-\infty)):=\left\{\widehat{\lambda}_{1 \pm}, \widehat{\lambda}_{2 \pm}\right\} \subset \mathbb{R}$, where $\widehat{\lambda}_{1 \pm}, \widehat{\lambda}_{2 \pm}$ are defined in $(22)$, and their eigenvalues satisfy the inequalities $\widehat{\lambda}_{1-}<0<\widehat{\lambda}_{1+}$, and $\widehat{\lambda}_{2-}<$ $0<\widehat{\lambda}_{2+}$.

Moreover, there is a real two-dimensional manifold $S$ containing the origin, such that any solution $Z$ of (19a) with $Z\left(\xi_{0}\right) \in S$ for any $\xi_{0}$, satisfy $Z(\xi) \rightarrow 0$ as $\xi \rightarrow-\infty$, and

$$
\liminf _{\xi \rightarrow-\infty} \frac{\log |Z(\xi)|}{\xi} \geq \mu_{-\infty}:=\max \left\{\widehat{\lambda}_{1-}, \widehat{\lambda}_{2-}\right\}>0,
$$

where $Z(\xi)=\left(z_{n}(\xi), z_{n}^{\prime}(\xi), z_{h}(\xi), z_{h}^{\prime}(\xi)\right)$.

(iii) There is a constant $C>0$ such that any solution $Z$ near the origin, but not on $S$ at $\xi=\xi_{0}$ can not satisfy $|Z(\xi)| \leq C$ for $\xi \leq \xi_{0}$.

Proof. This is a standard result in the theory of asymptotic behavior of ordinary differential equations [14, Chapter 13].

We have to analyze the sign of $\operatorname{Re}(\sigma(A))$ where $\sigma(A):=\left\{\lambda_{1_{+}}, \lambda_{1_{-}}, \lambda_{2+}, \lambda_{2-}\right\}$, and $\lambda_{1 \pm}, \lambda_{2 \pm}$ are defined as

$$
\lambda_{1 \pm}:=\frac{-c \pm \sqrt{c^{2}-4 \frac{D_{n}}{\tau_{n}}}}{2 D_{n}}, \lambda_{2 \pm}:=\frac{-c \pm \sqrt{c^{2}-4 D_{h}\left(\frac{1}{\tau_{h}}-\frac{1}{\tau_{h n}}\right)}}{2 D_{h}} .
$$

Obviously, if $\tau_{h n} \geq \tau_{h}$, then $c_{h n}^{*} \in \mathbb{R}$. Moreover, if $c>c_{n}^{*}>0$, then the eigenvalues $\lambda_{1 \pm}$ are negative real numbers. When $c>c_{h n}^{*}$, then the eigenvalues $\lambda_{2 \pm}$ are negative real numbers.

The eigenvalues of $\sigma(A+B(-\infty))$ are given by

$$
\widehat{\lambda}_{1 \pm}:=\frac{-c \pm \sqrt{c^{2}+4 D_{n} / \tau_{n}}}{2 D_{n}}, \quad \widehat{\lambda}_{2 \pm}:=\frac{-c \pm \sqrt{c^{2}+4 D_{h} / \tau_{h n}}}{2 D_{h}} .
$$

Whenever $0<\tau_{n}<\infty$, and $\tau_{h n}>0$, the eigenvalues $\hat{\lambda}_{1 \pm}, \widehat{\lambda}_{2 \pm}$ are real numbers and satisfy $\widehat{\lambda}_{1-}<0<\widehat{\lambda}_{1+}$, and $\widehat{\lambda}_{2-}<0<\widehat{\lambda}_{2+}$. 
Proof. of Theorem 3. Let $V=V\left(\xi ; V_{0}\right)$ be a solution of the Eq. (A.1). From Theorem 19, there exists a real 2-dimensional unstable manifold of the equilibrium $(1,0,0,0)$ denoted by $E^{u}$. In other words, for any $V_{0} \in E^{u}$, the trajectory $V=V\left(\xi ; V_{0}\right)$ satisfies $V=V\left(\xi ; V_{0}\right) \rightarrow(1,0,0,0)$ as $\xi \rightarrow-\infty$.

Let $z=\left(z_{n}, z_{h}\right)$ be a solution of Eq. (17). Since $\left(z_{n}, z_{h}\right):=\left(v_{n}-v_{K}, v_{h}\right)$, then $Z=\left(Z_{1}, Z_{2}, Z_{3}, Z_{4}\right)=\left(z_{n}, z_{n}^{\prime}, z_{h}, z_{h}^{\prime}\right)$, satisfies Eq. (19a), and $Z=\left(v_{n}-\right.$ $\left.v_{K}, v_{n}^{\prime}-v_{K}^{\prime}, v_{h}, v_{h}^{\prime}\right)=V-\left(v_{K}, v_{K}^{\prime}, 0,0\right)$.

Also, from (15), for any $\varepsilon>0$ there is a $\xi_{1} \in \mathbb{R}$ such that

$$
|Z(\xi)| \leq|V(\xi)-(1,0,0,0)|+\left|\left(v_{K}(\xi), v_{K}^{\prime}(\xi), 0,0\right)-(1,0,0,0)\right| \leq \varepsilon,
$$

for any $\xi \leq \xi_{1}$. Let $Z^{1}=Z\left(\xi_{1}\right)$ and consider the trajectory $Z=Z\left(\xi ; Z^{1}\right)$. Proposition 5 implies that $Z=Z\left(\xi ; Z^{1}\right)$ satisfies $|Z| \rightarrow 0$ as $\xi \rightarrow \infty$. Therefore, Eq. (15) guarantees that $V=Z+\left(v_{K}, v_{K}^{\prime}, 0,0\right) \rightarrow(0,0,0,0)$ as $\xi \rightarrow \infty$.

Next, we try to find necessary conditions for having a bifurcation from the trivial solution. Let us first pose the abstract framework of the problem.

A weak solution of

$$
L_{1} u_{1}=f_{1}, \quad L_{2} u_{2}=f_{2},
$$

is defined in the usual way, i.e. multiplying by a test function $\psi=\left(\psi_{1}, \psi_{2}\right) \in$ $H^{1}(\mathbb{R})^{2}$ and integrating on $\mathbb{R}$. Thus $\forall \psi=\left(\psi_{1}, \psi_{2}\right) \in H^{1}(\mathbb{R})^{2}$, we obtain

$$
\begin{aligned}
& \int_{\mathbb{R}} D_{n} u_{1}^{\prime} \psi_{1}^{\prime}-c u_{1}^{\prime} \psi_{1}+\frac{2}{\tau_{n}} v_{K} u_{1} \psi_{1}+\frac{1}{\tau_{n}} u_{1} \psi_{1} \\
& \quad+\int_{\mathbb{R}} D_{h} u_{2}^{\prime} \psi_{2}^{\prime}-c u_{2}^{\prime} \psi_{2}+\frac{1}{\tau_{h}} v_{K} u_{2} \psi_{2}+\frac{1}{\tau_{h}} u_{2} \psi_{2}=\int_{\mathbb{R}} f_{1} \psi_{1}+f_{2} \psi_{2}
\end{aligned}
$$

Let us set $H:=H^{1}(\mathbb{R})^{2}$ with $\|u\|_{H}:=\left(\left\|u_{1}\right\|^{2}+\left\|u_{2}\right\|^{2}\right)^{1 / 2}$ and define the bilinear form $a: H \times H \rightarrow \mathbb{R}$ given by

$$
\begin{aligned}
a(u, \psi):=\int_{\mathbb{R}}\left(D_{n} u_{1}^{\prime} \psi_{1}^{\prime}-c u_{1}^{\prime} \psi_{1}+\frac{2}{\tau_{n}} v_{K} u_{1} \psi_{1}+\frac{1}{\tau_{n}} u_{1} \psi_{1}\right) \\
+\int_{\mathbb{R}}\left(D_{h} u_{2}^{\prime} \psi_{2}^{\prime}-c u_{2}^{\prime} \psi_{2}+\frac{1}{\tau_{h}} v_{K} u_{2} \psi_{2}+\frac{1}{\tau_{h}} u_{2} \psi_{2}\right)
\end{aligned}
$$

for all $u=\left(u_{1}, u_{2}\right), \psi=\left(\psi_{1}, \psi_{2}\right) \in H$.

Theorem 6. Assume that $\min \left\{D_{n}, D_{h}\right\}>0$, and that $0<\tau_{n}, \tau_{h}<\infty$. Then, for all $f=\left(f_{1}, f_{2}\right) \in H^{*}$, the dual space of all linear continuous functional on $H$, there exists a unique $u=\left(u_{1}, u_{2}\right) \in H$ such that

$$
a(u, \psi)=\langle f, \psi\rangle_{H^{*}, H} \quad \text { for all } \quad \psi=\left(\psi_{1}, \psi_{2}\right) \in H,
$$

and

$$
\|u\|_{H} \leq C\|f\|_{H^{*}} .
$$


The constant $C$ can be chosen as $C:=1 / \min \left\{D_{n}, 1 / \tau_{n}, D_{h}, 1 / \tau_{h}\right\}$.

Moreover, if $f=\left(f_{1}, f_{2}\right) \in L^{2}(\mathbb{R})^{2}$, then $u=\left(u_{1}, u_{2}\right) \in H^{2}(\mathbb{R})^{2}$, and $\left(u_{1}, u_{1}^{\prime}, u_{2}, u_{2}^{\prime}\right) \rightarrow(0,0,0,0)$ as $|\xi| \rightarrow+\infty$.

Furthermore, if $f=\left(f_{1}, f_{2}\right) \in L^{2}(\mathbb{R})^{2} \cap C(\mathbb{R})^{2}$, then $u=\left(u_{1}, u_{2}\right) \in C^{2}(\mathbb{R})^{2} \cap$ $H^{2}(\mathbb{R})^{2}$, and $\|u\|_{H^{2}(\mathbb{R})^{2}} \leq C\|f\|_{L^{2}(\mathbb{R})^{2}}$.

Proof. The bilinear form $a$ is continuous and coercive. Indeed, by definition

$$
\begin{aligned}
a(u, u)=\int_{\mathbb{R}}\left(D_{n}\left(u_{1}^{\prime}\right)^{2}-\right. & \left.c u_{1}^{\prime} u_{1}+\frac{2}{\tau_{n}} v_{K} u_{1}^{2}+\frac{1}{\tau_{n}} u_{1}^{2}\right) \\
& +\int_{\mathbb{R}}\left(D_{h}\left(u_{2}^{\prime}\right)^{2}-c u_{2}^{\prime} u_{2}+\frac{1}{\tau_{h}} v_{K} u_{2}^{2}+\frac{1}{\tau_{h}} u_{2}^{2}\right),
\end{aligned}
$$

and since $\int_{R}^{R} u_{1}^{\prime} u_{1}=\left.\frac{1}{2} u_{1}^{2}\right|_{-R} ^{R}=\frac{1}{2}\left[u_{1}(R)^{2}-u_{1}(-R)^{2}\right] \rightarrow 0$ as $R \rightarrow \infty$ see $[9$, Corollary 8.9], we obtain

$$
\begin{aligned}
a(u, u) & =\int_{\mathbb{R}}\left(D_{n}\left(u_{1}^{\prime}\right)^{2}+\frac{2}{\tau_{n}} v_{K} u_{1}^{2}+\frac{1}{\tau_{n}} u_{1}^{2}+D_{h}\left(u_{2}^{\prime}\right)^{2}+\frac{1}{\tau_{h}} v_{K} u_{2}^{2}+\frac{1}{\tau_{h}} u_{2}^{2}\right) \\
& \geq \min \left\{D_{n}, \frac{1}{\tau_{n}}\right\}\left\|u_{1}\right\|^{2}+\min \left\{D_{h}, \frac{1}{\tau_{h}}\right\}\left\|u_{2}\right\|^{2} \\
& \geq \alpha\left(\left\|u_{1}\right\|^{2}+\left\|u_{2}\right\|^{2}\right)=\alpha\|u\|_{H}^{2}
\end{aligned}
$$

for all $u=\left(u_{1}, u_{2}\right) \in H$, for $\alpha:=\min \left\{D_{n}, 1 / \tau_{n}, D_{h}, 1 / \tau_{h}\right\}>0$. It follows that $\alpha\|u\|_{H}^{2} \leq a(u, u)=\langle f, u\rangle_{H^{*}, H} \leq\|f\|_{H^{*}}\|\| u \|_{H}$ and thus $\|u\|_{H} \leq C\|f\|_{H^{*}}$ with a constant $C$ dependent only on $\alpha$. The Lax-Milgram lemma completes this part of the proof.

By hypothesis $u \in H=H^{1}(\mathbb{R})^{2}$. First, note that if $f, u^{\prime} \in L^{2}(\mathbb{R})^{2}$, then by definition of $H^{1}, u^{\prime} \in H^{1}(\mathbb{R})^{2}$, therefore $u \in H^{2}(\mathbb{R})^{2}$. Moreover, by Brézis [9, Corollary 8.9], $u_{1}, u_{2}, u_{1}^{\prime}, u_{2}^{\prime} \rightarrow 0$ as $|\xi| \rightarrow+\infty$.

Due to $u \in H^{2}(\mathbb{R})^{2}$, then $u, u^{\prime} \in C(\mathbb{R})^{2}$. Furthermore, if $f=\left(f_{1}, f_{2}\right) \in$ $L^{2}(\mathbb{R})^{2} \cap C(\mathbb{R})^{2}$, since $u, u^{\prime}, v_{K}$, and $f$ are continuous on $\mathbb{R}$ we get $u^{\prime \prime} \in C(\mathbb{R})^{2}$, and therefore $u \in C^{2}(\mathbb{R})^{2}$.

Finally, taking into account that $u$ is a classical solution, we get

$$
\left\|u^{\prime \prime}\right\|_{L^{2}(\mathbb{R})^{2}} \leq C\left(\|u\|_{H^{1}(\mathbb{R})^{2}}+\|f\|_{L^{2}(\mathbb{R})^{2}}\right) \leq C\|f\|_{L^{2}(\mathbb{R})^{2}},
$$

which completes the proof.

Corollary 7. Assume that $f_{j} \rightarrow f$ in $L^{2}(\mathbb{R})^{2}$. Let $u_{j}, j \geq 0$, be such that $a\left(u_{j}, \psi\right)=\left\langle f_{j}, \psi\right\rangle_{H^{*}, H}$, for all $\psi=\left(\psi_{1}, \psi_{2}\right) \in H$, . Then $u_{j} \rightarrow u$ in $H^{2}(\mathbb{R})^{2}$

Proof. It follows from theorem 6 taking $g_{j}=f_{j}-f$ and $v_{j}=u_{j}-u$.

Let $f=\left(f_{1}, f_{2}\right), f=f(z)$, denote the nonlinearity of $(17)$, i.e.

$$
\begin{aligned}
f_{1}\left(z_{n}, z_{h}\right) & :=\frac{1}{\tau_{n}}\left(2-z_{n}-z_{h}\right) z_{n}+\left(\frac{1}{\tau_{h n}}-\frac{1}{\tau_{n}} v_{K}\right) z_{h}, \\
f_{2}\left(z_{n}, z_{h}\right) & :=\frac{1}{\tau_{h}}\left(2-z_{n}-z_{h}\right) z_{h}-\frac{1}{\tau_{h n}} z_{h} .
\end{aligned}
$$


We will say that $z=\left(z_{n}, z_{h}\right) \in H$ is a weak solution of (17) if and only if (17) is satisfied in a weak sense, which means that

$$
a(z, \psi)=\int_{\mathbb{R}} f_{1}\left(z_{n}, z_{h}\right) \psi_{1}+f_{2}\left(z_{n}, z_{h}\right) \psi_{2}
$$

for all $\psi=\left(\psi_{1}, \psi_{2}\right) \in H$.

The following result ensures that a weak solution is a classical solution, and provide the rates at $\pm \infty$.

Theorem 8. Assume that $\min \left\{D_{n}, D_{h}\right\}>0$, and that $0<\tau_{n}, \tau_{h}<\infty$. Assume that $\tau_{h n}>\tau_{h}$ and $c>\max \left\{c_{n}^{*}, c_{h n}^{*}\right\}$, where $c_{n}^{*}, c_{h n}^{*}$ are defined in (10a), (16) respectively. Let $z=\left(z_{n}, z_{h}\right) \in H$ be a weak solution of (17).

Then the following holds:

i) $z=\left(z_{n}, z_{h}\right) \in C^{2}(\mathbb{R})^{2} \cap H^{2}(\mathbb{R})^{2}$, and $\|z\|_{H^{2}(\mathbb{R})^{2}} \leq C\|z\|_{L^{2}(\mathbb{R})^{2}}$.

ii) Let $Z=\left(z_{n}, z_{n}^{\prime}, z_{h}, z_{h}^{\prime}\right)$, then

$$
\limsup _{\xi \rightarrow+\infty} \frac{\log |Z(\xi)|}{\xi} \leq \mu_{+\infty}<0, \quad \text { and } \quad \liminf _{\xi \rightarrow-\infty} \frac{\log |Z(\xi)|}{\xi} \geq \mu_{-\infty}>0
$$

where $\mu_{+\infty}:=\max \sigma(A)=\left\{\lambda_{1+}, \lambda_{1-}, \lambda_{2+}, \lambda_{2-}\right\}, \mu_{-\infty}:=\max \left\{\widehat{\lambda}_{1-}, \widehat{\lambda}_{2-}\right\}$, and $\lambda_{1 \pm}, \lambda_{2 \pm}, \widehat{\lambda}_{1-}, \widehat{\lambda}_{2-}$ are defined in (21), (22) respectively.

Proof. i) By Morrey's Theorem, see [9], there is a constant $C$ such that

$$
\left|z_{i}(x)-z_{i}(y)\right| \leq C\left\|\nabla z_{i}\right\|_{L^{2}(\mathbb{R})}|x-y|^{1 / 2}, \quad \text { a.e. } \quad x, y \in \mathbb{R}, \quad i \in\{n, h\} .
$$

Moreover, due to $z \in H$, we get that $\left(z_{n}, z_{h}\right) \rightarrow(0,0)$ as $|\xi| \rightarrow+\infty$. Therefore, applying Theorem 6 , we complete this part of the the proof.

ii) From part i) we get that $\left(z_{n}, z_{n}^{\prime}, z_{h}, z_{h}^{\prime}\right) \rightarrow(0,0,0,0)$ as $|\xi| \rightarrow+\infty$ and therefore, applying Proposition 5 we conclude the proof.

Lemma 9. Assume that $\tau_{h n}>\tau_{h}$ and $c>\max \left\{c_{n}^{*}, c_{h n}^{*}\right\}$, where $c_{n}^{*}, c_{h n}^{*}$ are defined in (10a), (16) respectively. Let $\left(v_{n}, v_{h}\right)$ be a nonegative solution of (12), with $v_{n} \not \equiv 0, v_{h} \not \equiv 0$. Then,

$$
0<v_{n} \leq \max \left\{1, \tau_{n} / \tau_{h n}\right\}, \quad \text { and } \quad 0<v_{h} \leq 1-\tau_{h} / \tau_{h n} .
$$

Remark 10. Since $\left(v_{n}, v_{h}\right)$ is a nonnegative solution of Eq. (12), then $\left(z_{n}, z_{h}\right)=$ $\left(v_{n}-v_{K}, v_{h}\right)$ is a solution of Eq. (17). Therefore, Lemma 9 implies that

$$
-1 \leq z_{n} \leq \max \left\{1, \tau_{n} / \tau_{h n}\right\}, \quad 0 \leq z_{h} \leq 1-\tau_{h} / \tau_{h n} .
$$

Proof. of Lemma 9. Let $\left(v_{n}, v_{h}\right)=\left(z_{n}+v_{K}, z_{h}\right)$ be a nonnegative solution of (12) with $v_{n} \neq 0, v_{h} \neq 0$. Note that $v_{n}, v_{h}$ can only have simple zeros, in the sense that if $v_{n}(x)=0$, then $v_{n}^{\prime}(x) \neq 0$. Therefore $v_{n}, v_{h}>0$ on $\mathbb{R}$. 
Let $x_{n}, x_{h} \in \mathbb{R}$ be such that $v_{n}\left(x_{n}\right)=\max _{\mathbb{R}} v_{n}>0, v_{h}\left(x_{h}\right)=\max _{\mathbb{R}} v_{h}>0$. Then, $v_{i}^{\prime}\left(x_{i}\right)=0, v_{i}^{\prime \prime}\left(x_{i}\right) \leq 0, i \in\{n, h\}$ and by (12) we obtain

$$
-D_{h} v_{h}^{\prime \prime}\left(x_{h}\right)=\frac{1}{\tau_{h}}\left(1-v_{n}\left(x_{h}\right)-v_{h}\left(x_{h}\right)\right) v_{h}\left(x_{h}\right)-\frac{1}{\tau_{h n}} v_{h}\left(x_{h}\right) \geq 0 .
$$

Dividing by $v_{h}\left(x_{h}\right)>0$, we get $1 / \tau_{h}-1 / \tau_{h n} \geq\left(v_{n}\left(x_{h}\right)+v_{h}\left(x_{h}\right)\right) / \tau_{h} \geq v_{h}\left(x_{h}\right) / \tau_{h}$ and the inequalities related to $v_{h}$ in (31) are attained.

Likewise $-D_{n} v_{n}^{\prime \prime}\left(x_{n}\right)=\frac{1}{\tau_{n}}\left(1-v_{n}\left(x_{n}\right)-v_{h}\left(x_{n}\right)\right) v_{n}\left(x_{n}\right)+\frac{1}{\tau_{h n}} v_{h}\left(x_{n}\right) \geq 0$, and therefore $\frac{1}{\tau_{n}}\left(1-v_{n}\left(x_{n}\right)\right) v_{n}\left(x_{n}\right)+\left(\frac{1}{\tau_{h n}}-\frac{1}{\tau_{n}} v_{n}\left(x_{n}\right)\right) v_{h}\left(x_{n}\right) \geq 0$.

If $v_{n}\left(x_{n}\right) \geq \tau_{n} / \tau_{h n}$ then

$$
\left(1-v_{n}\left(x_{n}\right)\right) v_{n}\left(x_{n}\right) / \tau_{n} \geq\left(v_{n}\left(x_{n}\right) / \tau_{n}-1 / \tau_{h n}\right) v_{h}\left(x_{n}\right) \geq 0,
$$

and dividing by $v_{n}\left(x_{n}\right)>0$, we get $v_{n}\left(x_{n}\right) \leq 1$. Also $v_{n}\left(x_{n}\right) \leq \tau_{n} / \tau_{h n}$, and the inequalities related to $v_{n}$ in (31) are attained, completing the proof.

Finally, we may prove our main result in this section that states necessary conditions for having solutions bifurcating from the solution $\left(v_{K}, 0\right)$ and positive in the second component, i.e. persistent finite amplitude solitons composed of cells with an hypoxic phenotype. Technically we use classical bifurcation methods. The convergence in any bounded interval is clear, and also that the limit function is a classical solution of the limit problem in all $\mathbb{R}$. The difficulty comes when trying to prove that the convergence is, in fact, in all $\mathbb{R}$ and as a consequence, that the limit function is non-trivial.

To achieve prove that, we need uniform estimates of the $H^{1}$ norms in the exterior of bounded intervals. In particular, we have to control the behavior at $\pm \infty$. We overcome this difficulty by using perturbation theory in this framework.

The interesting asymptotic behavior is obtained when $\tau_{h n}>\tau_{h}$, and $c>$ $\max \left\{c_{n}^{*}, c_{h n}^{*}\right\}$, where $c_{n}^{*}, c_{h n}^{*}$ are defined in (10a) and (16) respectively, see Appendix and Proposition 5.

Theorem 11. Let $\left(\Lambda_{j}, z_{j}\right) \in \mathbb{R}^{3} \times H$, be a sequence solving (17), with $\Lambda_{j}:=$ $\left(\tau_{n, j}, \tau_{h, j}, \tau_{h n, j}\right) \in \mathbb{R}^{3}$, and $z_{j}=\left(z_{n, j}, z_{h, j}\right) \in H$ with $z_{h, j}>0$. Assume that $\left\|z_{j}\right\|_{L^{2}(\mathbb{R})^{2}} \rightarrow 0$. Assume also that $\lim _{\sup _{j \rightarrow \infty}} \tau_{h, j}=: \bar{\tau}_{h}>0$, that $\lim _{j \rightarrow \infty} \tau_{h n, j}=:$ $\bar{\tau}_{h n}>0$, that $\tau_{h n, j}>\tau_{h, j}$, and that $c>\max \left\{c_{n, j}^{*}, c_{h n, j}^{*}\right\}$, for some subsequence again denoted by $\tau_{h n, j}$. Then,

$$
\frac{2}{\bar{\tau}_{h}}-\frac{1}{\bar{\tau}_{h n}} \in \sigma\left(L_{2}\right) .
$$

Moreover, at least for a subsequence,

$$
\frac{z_{h, j}}{\left\|z_{h, j}\right\|_{L^{2}(\mathbb{R})}} \rightarrow \Phi_{h}>0 \quad \text { in } \quad H^{1}(\mathbb{R}), \quad\left\|\Phi_{h}\right\|_{L^{2}(\mathbb{R})}=1,
$$


and the function $\Phi_{h} \in C^{2}(\mathbb{R}) \cap H^{2}(\mathbb{R})$ satisfies

$$
-D_{h} \Phi_{h}^{\prime \prime}-c \Phi_{h}^{\prime}+\frac{1}{\bar{\tau}_{h}} v_{K} \Phi_{h}=\left(\frac{1}{\bar{\tau}_{h}}-\frac{1}{\bar{\tau}_{h n}}\right) \Phi_{h} .
$$

Proof. Let us choose a subsequence such that $\left(\tau_{n, j}, \tau_{h, j}, \tau_{h n, j},\left\|z_{j}\right\|_{L^{2}(\mathbb{R})^{2}}\right) \rightarrow$ $\left(\bar{\tau}_{n}, \bar{\tau}_{h}, \bar{\tau}_{h n}, 0\right)$. Moreover, $\left\|z_{j}\right\|_{H} \leq C\left\|z_{j}\right\|_{L^{2}(\mathbb{R})^{2}} \rightarrow 0$. Also, see Theorem 6 and Remark 10, $\left\|z_{j}\right\|_{H^{2}(\mathbb{R})^{2}} \leq C\left\|z_{j}\right\|_{L^{2}(\mathbb{R})^{2}} \rightarrow 0$.

Let $\Phi_{h j}:=z_{h, j} /\left\|z_{h, j}\right\|_{L^{2}(\mathbb{R})} \geq 0$. Then $\left\|\Phi_{h j}\right\|_{L^{2}(\mathbb{R})}=1$ and

$$
\left\|\Phi_{h j}\right\|_{L^{\infty}(\mathbb{R})} \leq\left\|\Phi_{h j}\right\|_{H^{1}(\mathbb{R})} \leq C, \quad \text { and } \quad\left\|\Phi_{h j}\right\|_{H^{2}(\mathbb{R})} \leq C .
$$

From the Kolmogorov-Riesz-Fréchet's Theorem, see [9, Theorem 4.26 and Corollary 4.27], for any bounded interval $I$ fixed, there exists a subsequence $\Phi_{h j} \rightarrow \Phi_{h}$ in $H^{1}(I)$. If moreover, for any $\varepsilon>0$, there exists a bounded interval $I=I(\varepsilon)$ such that

$$
\left\|\Phi_{h j}\right\|_{H^{1}(\mathbb{R} \backslash I)}<\varepsilon
$$

then, there exists a subsequence such that $\Phi_{h j} \rightarrow \Phi_{h}$ in $H^{1}(\mathbb{R})$.

Let us fix $R_{0}>0$, then $\Phi_{h j} \rightarrow \Phi_{h}$ in $H^{1}\left(-R_{0}, R_{0}\right)$, where $\Phi_{h}$ depends on $R_{0}$, (we shall denote it by $\Phi_{h, R_{0}}$ when we need to remark this dependence). In order to achieve (34), let us divide Eq. (17b) by $\left\|z_{2 j}\right\|_{L^{2}(\mathbb{R})}$, then we obtain

$$
L_{2} \Phi_{h j}=\left(2-z_{n, j}-z_{h, j}\right) \Phi_{h j} / \tau_{h, j}-P h i_{h j} / \tau_{h n, j}
$$

Let $\zeta_{j}=\left(\Phi_{h j}, \Phi_{h j}^{\prime}\right)$ be the solution of the associated IVP

$$
\begin{aligned}
\frac{d \zeta}{d \xi} & =A \zeta+B(\xi) \zeta+\left(A_{j}-A\right) \zeta+\left[B_{j}(\xi)-B(\xi)\right] \zeta \\
\zeta(0) & =\left(\Phi_{h j}(0), \Phi_{h j}^{\prime}(0)\right),
\end{aligned}
$$

where

$$
\begin{gathered}
A_{j}:=\left(\begin{array}{cc}
1 \\
\frac{1}{D_{h}}\left(\frac{1}{\tau_{h n, j}}-\frac{1}{\tau_{h, j}}\right)-\frac{c}{D_{h}}
\end{array}\right), A:=\left(\begin{array}{cc}
1 \\
\frac{1}{D_{h}}\left(\frac{1}{\bar{\tau}_{h n}}-\frac{1}{\bar{\tau}_{h}}\right) & -\frac{c}{D_{h}}
\end{array}\right), \\
B_{j}(\xi):=\left(\begin{array}{cc}
0 & 0 \\
\frac{v_{K}}{D_{h} \tau_{h, j}}+\frac{z_{n, j}+z_{h, j}}{D_{h} \tau_{h, j}} & 0
\end{array}\right), \quad B(\xi):=\left(\begin{array}{cc}
0 & 0 \\
\frac{v_{K}}{D_{h} \bar{\tau}_{h}} & 0
\end{array}\right),
\end{gathered}
$$

and $A_{j} \rightarrow A$ as $j \rightarrow \infty, B_{j}(\xi) \rightarrow B(\xi)$ as $j \rightarrow \infty$ for each $\xi \in \mathbb{R}$. Moreover, using (15) and since $\left(z_{n, j}, z_{h, j}\right) \rightarrow(0,0)$ as $j \rightarrow \infty$, see Theorem 8 , we get

$$
\begin{aligned}
& \lim _{\xi \rightarrow+\infty} B_{j}(\xi)=: \quad B_{j}(+\infty)=\left(\begin{array}{ll}
0 & 0 \\
0 & 0
\end{array}\right), \\
& \lim _{\xi \rightarrow-\infty} B_{j}(\xi)=: \quad B_{j}(-\infty)=\left(\begin{array}{cc}
0 & 0 \\
1 /\left(D_{h} \tau_{h, j}\right) & 0
\end{array}\right)
\end{aligned}
$$


Eq. (36) is an homogeneous linear problem. From classical perturbation theory (see [14]), we get the asymptotic behavior

$$
\limsup _{\xi \rightarrow+\infty} \frac{\log \left|\left(\Phi_{h j}(\xi), \Phi_{h j}^{\prime}(\xi)\right)\right|}{\xi} \leq\left(\lambda_{2+}\right)+\varepsilon<0,
$$

and

$$
\liminf _{\xi \rightarrow+\infty} \frac{\log \left|\left(\Phi_{h j}(-\xi), \Phi_{h j}^{\prime}(-\xi)\right)\right|}{-\xi} \geq\left(\widehat{\lambda}_{2+}\right)-\varepsilon>0 .
$$

where $\lambda_{2+}, \widehat{\lambda}_{2+}$ are defined in (21),(22) respectively.

Therefore (34) is accomplished, $\Phi_{h j} \rightarrow \Phi_{h}$ in $H^{1}(\mathbb{R})$ and $\Phi_{h}$ solves (32). In particular $\left\|\Phi_{h}\right\|_{L^{2}(\mathbb{R})}=1$ which implies $\Phi_{h} \neq 0$, and then $\Phi_{h} \supsetneqq 0$. The Maximum Principle implies that $\Phi_{h}>0$.

Multiplying (32) by $\Phi_{h}$ and integrating on $\mathbb{R}$ we obtain

$$
D_{h} \int_{\mathbb{R}}\left(\Phi_{h}^{\prime}\right)^{2}+\frac{1}{\bar{\tau}_{h}} \int_{\mathbb{R}} v_{K} \Phi_{h}^{2}=\left(\frac{1}{\bar{\tau}_{h}}-\frac{1}{\bar{\tau}_{h n}}\right) \int_{\mathbb{R}} \Phi_{h}^{2},
$$

so $1 / \bar{\tau}_{h}-1 / \bar{\tau}_{h n} \geq 0$ which completes the proof.

Remark 12. The asymptotic behavior provided by (39a) is un upper bound. In the same sprit as Proposition 5, (ii), there exists a real one-dimensional manifold $S_{1}$ containing the origin, such that any solution $\left(\Phi_{h}, \Phi_{h}^{\prime}\right)$ of

$$
\frac{d \zeta}{d \xi}=A \zeta+B(\xi) \zeta, \quad \zeta(0)=\left(\Phi_{h}(0), \Phi_{h}^{\prime}(0)\right),
$$

where $A, B$ are defined in (37), and with $\left(\Phi_{h}\left(\xi_{0}\right), \Phi_{h}^{\prime}\left(\xi_{0}\right)\right) \in S_{1}$ for any $\xi_{0}$, satisfy $\Phi_{h}(\xi), \Phi_{h}^{\prime}(\xi) \rightarrow 0$ as $\xi \rightarrow \infty$, and

$$
\limsup _{\xi \rightarrow \infty} \frac{\log \left|\left(\Phi_{h j}(\xi), \Phi_{h j}^{\prime}(\xi)\right)\right|}{\xi} \leq\left(\lambda_{2-}\right)+\varepsilon<0,
$$

where $\lambda_{2-}$ is defined in (21).

From a theoretical point of view, the hypoxic front wave can have two 'components' when $\xi \rightarrow \infty$, one corresponding to the biggest eigenvalue $\lambda_{2+}$, and the other one to the smallest eigenvalue $\lambda_{2-}$. From a quantitative point of view, the component corresponding to the biggest eigenvalue $\lambda_{2+}$, is much bigger than the component corresponding to the smallest eigenvalue $\lambda_{2-}$. The above result states that the speed wave of the component of the hypoxic front wave corresponding to the smallest eigenvalue converges to $c_{h n}^{*}$. 
Corollary 13. Let $\left(\Lambda_{j}, z_{j}\right) \in \mathbb{R}^{4} \times H$, be a sequence solving (17), with $\Lambda_{j}:=$ $\left(\tau_{n, j}, \tau_{h, j}, \tau_{h n, j}, c_{j}\right) \in \mathbb{R}^{4}$, and $z_{j}=\left(z_{n, j}, z_{h, j}\right) \in H$ with $z_{h, j}>0$. Assume that $\left\|z_{j}\right\|_{L^{2}(\mathbb{R})^{2}} \rightarrow 0$. Assume also that $\limsup _{j \rightarrow \infty} \tau_{h, j}=: \bar{\tau}_{h}>0$, that $\lim _{j \rightarrow \infty} \tau_{h n, j}=:$ $\bar{\tau}_{h n}>0$, that $\tau_{h n, j}>\tau_{h, j}$, that $c_{j}>\max \left\{c_{n, j}^{*}, c_{h n, j}^{*}\right\}$, and that $\lim _{j \rightarrow \infty} c_{j}=$ : $\bar{c}>0$, for some subsequence again denoted by $\Lambda_{j}$.

Let us keep the notation of Theorem 11.

Assume that for any $\varepsilon>0$ there exists a $j_{0}$ such that if $j \geq j_{0}$ then

$$
\limsup _{\xi \rightarrow \infty} \frac{\log \left|\left(\Phi_{h j}(\xi), \Phi_{h j}^{\prime}(\xi)\right)\right|}{\xi} \leq\left(\lambda_{2-, j}\right)+\varepsilon<0,
$$

where $\lambda_{2-, j}$ are defined in (21) for $\left(\tau_{h}, \tau_{h n}, c\right)=\left(\tau_{h, j}, \tau_{h n, j}, c_{j}\right)$, then

$$
\bar{c}=c_{h n}^{*}=2 \sqrt{D_{h}\left(\frac{1}{\bar{\tau}_{h}}-\frac{1}{\bar{\tau}_{h n}}\right)} .
$$

Proof. By hypothesis we have that $c_{j}>\max \left\{c_{n, j}^{*}, c_{h n, j}^{*}\right\} \geq c_{h n, j}^{*}$. We shall argue by contradiction, assuming that $\bar{c}>c_{h n}^{*}$. By definition of $\lambda_{2-}$, see (21), we can state that $\lambda_{2-}<-\bar{c} / 2 D_{h}$, therefore, taking into account (41), there exists some $\varepsilon>0$ such that

$$
\limsup _{\xi \rightarrow+\infty} \frac{\log \left|\left(\Phi_{h}(\xi), \Phi_{h}^{\prime}(\xi)\right)\right|}{\xi} \leq-\frac{\bar{c}}{2 D_{h}}-\varepsilon<0,
$$

Let $\varphi:=e^{\alpha \xi} \Phi_{h}$ for some $\alpha$ to be determined later. Differentiating twice, substituting into the equation (32), and rearranging terms we can write

$$
-D_{h} \varphi^{\prime \prime}+\left(2 D_{h} \alpha-\bar{c}\right) \varphi^{\prime}+\left(-D_{h} \alpha^{2}+\bar{c} \alpha+v_{K} / \bar{\tau}_{h}\right) \varphi=\sigma \varphi, \text { for } \xi \in \mathbb{R},
$$

where $\sigma=1 / \bar{\tau}_{h}-1 / \bar{\tau}_{h n}$. Choosing $\alpha:=\bar{c} / 2 D_{h}$ we get

$$
\limsup _{\xi \rightarrow+\infty} \frac{\log \left|\left(\varphi(\xi), \varphi^{\prime}(\xi)\right)\right|}{\xi} \leq-\varepsilon<0,
$$

therefore $\varphi \in H^{1}(\mathbb{R})$ and $-D_{h} \varphi^{\prime \prime}+\left(\frac{\bar{c}^{2}}{4 D_{h}}+\frac{1}{\bar{\tau}_{h}} v_{K}\right) \varphi=\sigma \varphi$, for $\xi \in \mathbb{R}$. We can consider this as a self-adjoint problem in $L^{2}(\mathbb{R})$, regardless of the original space, so that any eigenvalue must be real. The smallest eigenvalue is

$$
\sigma=\inf _{\varphi \in H^{1}(\mathbb{R}): \int_{\mathbb{R}} \varphi^{2}=1}\left\{D_{h} \int_{\mathbb{R}}\left(\varphi^{\prime}\right)^{2}+\int_{\mathbb{R}}\left(\frac{\bar{c}^{2}}{4 D_{h}}+\frac{1}{\bar{\tau}_{h}} v_{K}\right) \varphi^{2}\right\} \geq \frac{\bar{c}^{2}}{4 D_{h}},
$$

and therefore $\sigma \geq \bar{c}^{2} / 4 D_{h}$. Taking into account that $\sigma=1 / \bar{\tau}_{h}-1 / \bar{\tau}_{h n}$, we obtain $1 / \bar{\tau}_{h}-1 / \bar{\tau}_{h n} \geq \bar{c}^{2} / 4 D_{h}$ or equivalently, that $\bar{c} \leq 2 \sqrt{D_{h}\left(1 / \tau_{h}-1 / \tau_{h n}\right)}=$ : $c_{h n}^{*}$, which contradicts the hypothesis.

For the first component, the following result holds 
Corollary 14. If the hypothesis of Theorem 11, hold, then

$$
\frac{z_{n, j}}{\left\|z_{j}\right\|_{L^{2}(\mathbb{R})^{2}}} \rightarrow \Phi_{n} \quad \text { in } \quad H^{1}(\mathbb{R})
$$

where $\|z\|_{L^{2}(\mathbb{R})^{2}}=\left(\left\|z_{n}\right\|_{L^{2}(\mathbb{R})^{2}}^{2}+\left\|z_{h}\right\|_{L^{2}(\mathbb{R})^{2}}^{2}\right)^{1 / 2}$.

Moreover, $\Phi_{n} \in C^{2}(\mathbb{R}) \cap H^{2}(\mathbb{R})$ is such that $\Phi_{n} \neq 0$, and for some $\theta \in[0,1)$

$$
-D_{n} \Phi_{n}^{\prime \prime}-c \Phi_{n}^{\prime}+\frac{2}{\bar{\tau}_{n}} v_{K} \Phi_{n}=\frac{1}{\bar{\tau}_{n}} \Phi_{n}+\theta\left(\frac{1}{\bar{\tau}_{h n}}-\frac{1}{\bar{\tau}_{n}} v_{K}\right) \Phi_{h} .
$$

Proof. Let $\Phi_{n j}:=z_{n, j} /\left\|z_{j}\right\|_{L^{2}(\mathbb{R})^{2}}$, where $\|z\|_{L^{2}(\mathbb{R})^{2}}^{2}=\left\|z_{n}\right\|_{L^{2}(\mathbb{R})^{2}}^{2}+\left\|z_{h}\right\|_{L^{2}(\mathbb{R})^{2}}^{2}$. Then $\left\|\Phi_{n j}\right\|_{L^{2}(\mathbb{R})} \leq 1$ and

$$
\left\|\Phi_{n j}\right\|_{L^{\infty}(\mathbb{R})} \leq\left\|\Phi_{n j}\right\|_{H^{1}(\mathbb{R})} \leq C, \quad \text { and } \quad\left\|\Phi_{n j}\right\|_{H^{2}(\mathbb{R})} \leq C .
$$

From the Kolmogorov-Riesz-Fréchet's Theorem, see Brézis [9, Theorem 4.26 and Corollary 4.27], for any bounded interval $I$ fixed, there exists a subsequence $\Phi_{n j} \rightarrow \Phi_{n}$ in $H^{1}(I)$. Let us fix $R_{0}>0$, then $\Phi_{n j} \rightarrow \Phi_{n, R_{0}}$ in $H^{1}\left(-R_{0}, R_{0}\right)$.

Obviously $\left\|z_{h, j}\right\|_{L^{2}(\mathbb{R})} /\left\|z_{j}\right\|_{L^{2}(\mathbb{R})^{2}} \leq 1$. Then, at least for a subsequence, $\frac{\left\|z_{h, j}\right\|_{L^{2}(\mathbb{R})}}{\left\|z_{j}\right\|_{L^{2}(\mathbb{R})^{2}}} \rightarrow \theta \in[0,1]$, and therefore, $\left\|\Phi_{n, j}\right\|_{L^{2}(\mathbb{R})}=\frac{\left\|z_{1 j}\right\|_{L^{2}(\mathbb{R})}}{\left\|z_{j}\right\|_{L^{2}(\mathbb{R})^{2}}} \rightarrow \sqrt{1-\theta^{2}}$.

Dividing by $\left\|z_{j}\right\|_{L^{2}(\mathbb{R})}$ the first equation of (17), we obtain

$$
L_{1} \Phi_{n j}=\frac{1}{\tau_{n, j}}\left(2-z_{n, j}-z_{h, j}\right) \Phi_{n j}+\left(\frac{1}{\tau_{h n, j}}-\frac{v_{K}}{\tau_{n, j}}\right) \theta \Phi_{h j} .
$$

Let now $\zeta=\left(\Phi_{n j}, \Phi_{n j}^{\prime}\right)$ be the solution of the associated IVP

$$
\frac{d \zeta}{d \xi}=A \zeta+B(\xi) \zeta+F(\xi)+\left(A_{j}-A\right) \zeta+\left[B_{j}(\xi)-B(\xi)\right] \zeta+\left[F_{j}(\xi)-F(\xi)\right],
$$

with $\zeta(0)=\left(\Phi_{n j}(0), \Phi_{n j}^{\prime}(0)\right)$, where

$$
\begin{aligned}
& A_{j}:=\left(\begin{array}{cc}
0 & 1 \\
-\frac{1}{D_{n} \tau_{n, j}} & -\frac{c}{D_{n}}
\end{array}\right), A:=\left(\begin{array}{cc}
0 & 1 \\
-\frac{1}{D_{n} \bar{\tau}_{n}} & -\frac{c}{D_{n}}
\end{array}\right), \\
& B_{j}(\xi):=\left(\begin{array}{cc}
0 & 0 \\
\frac{2 v_{K}}{D_{n} \tau_{n, j}}+\frac{z_{n, j}+z_{h, j}}{D_{n} \tau_{n, j}} & 0
\end{array}\right), \quad B(\xi):=\left(\begin{array}{cc}
0 & 0 \\
\frac{2 v_{K}}{D_{n} \bar{\tau}_{n}} & 0
\end{array}\right), \\
& F_{j}(\xi):=\left(\theta\left(\frac{v_{K}}{D_{n} \tau_{n, j}}-\frac{1}{\tau_{h n, j}}\right) \Phi_{h j}\right), F(\xi):=\left(\theta\left(\frac{v_{K}}{D_{n} \bar{\tau}_{n}}-\frac{1}{\bar{\tau}_{h n}}\right) \Phi_{h}\right),
\end{aligned}
$$


see Theorem 8 , we get

$$
\begin{aligned}
& \lim _{\xi \rightarrow+\infty} B_{j}(\xi)=: \quad B(+\infty)=\left(\begin{array}{ll}
0 & 0 \\
0 & 0
\end{array}\right), \\
& \lim _{\xi \rightarrow-\infty} B_{j}(\xi)=: \quad B_{j}(-\infty)=\left(\begin{array}{cc}
0 & 0 \\
2 /\left(D_{n} \tau_{n, j}\right) & 0
\end{array}\right) .
\end{aligned}
$$

From (39), we get

$$
\limsup _{\xi \rightarrow+\infty} \frac{\log \left|F_{j}(\xi)\right|}{\xi} \leq\left(\lambda_{2+}\right)+\varepsilon<0
$$

and

$$
\liminf _{\xi \rightarrow+\infty} \frac{\log \left|F_{j}(\xi)\right|}{-\xi} \geq\left(\widehat{\lambda}_{2+}\right)-\varepsilon>0,
$$

where $\lambda_{i \pm}, \widehat{\lambda}_{2+}$, are defined in (21),(22) respectively.

Eq. (49) is a non-homogeneous linear problem. We get its asymptotic behavior from the variation of parameters formula,

$$
\limsup _{\xi \rightarrow+\infty} \frac{\log \left|\left(\Phi_{n j}(\xi), \Phi_{n j}^{\prime}(\xi)\right)\right|}{\xi} \leq \max \left\{\lambda_{1+}, \lambda_{2+}\right\}+\varepsilon<0,
$$

and

$$
\liminf _{\xi \rightarrow+\infty} \frac{\log \left|\left(\Phi_{n j}(-\xi), \Phi_{n j}^{\prime}(-\xi)\right)\right|}{-\xi} \geq \min \left\{\widehat{\lambda}_{1+}, \widehat{\lambda}_{2+}\right\}-\varepsilon>0 .
$$

where $\widehat{\lambda}_{i+}, \lambda_{i+}, i=1,2$, are defined in (21),(22) respectively. Then $\Phi_{n j} \rightarrow \Phi_{n}$ in $H^{1}(\mathbb{R})$ and $\Phi_{n}$ solves $(46)$. If $\theta=1$ then $\left\|\Phi_{n}\right\|_{L^{2}(\mathbb{R})^{2}}=\sqrt{1-\theta^{2}}=0$ which contradicts (46), due to $\left\|\Phi_{h}\right\|_{L^{2}(\mathbb{R})^{2}}=1$, so $0 \leq \theta<1$ and $\Phi_{n} \neq 0$.

\section{Do ghost waves exist in three spatial dimensions}

A very interesting question relates to the existence of evanescent "ghost" waves driving tumor growth, in anisotropic three-dimensional spaces.

In general, the theoretical problem of existence and stability of travelling waves in higher-dimensional scenarios is not simple [8].

Our results, both analytical and numerical, are immediately applicable to flat three-dimensional plane-waves, i.e. waves with uniform profiles along two transversal directions. The general extension of our results to other types of solitary waves, such as radial solitary waves, is not trivial with few theoretical results available. However, in general, this type of reaction-diffusion equations displays the same type of dynamics independently on the spatial dimension (see e.g. Pérez-García et al. [41], Pérez-García and Pérez-Romasanta [42] for examples in simpler contexts) even when formally it is not possible to prove that. 


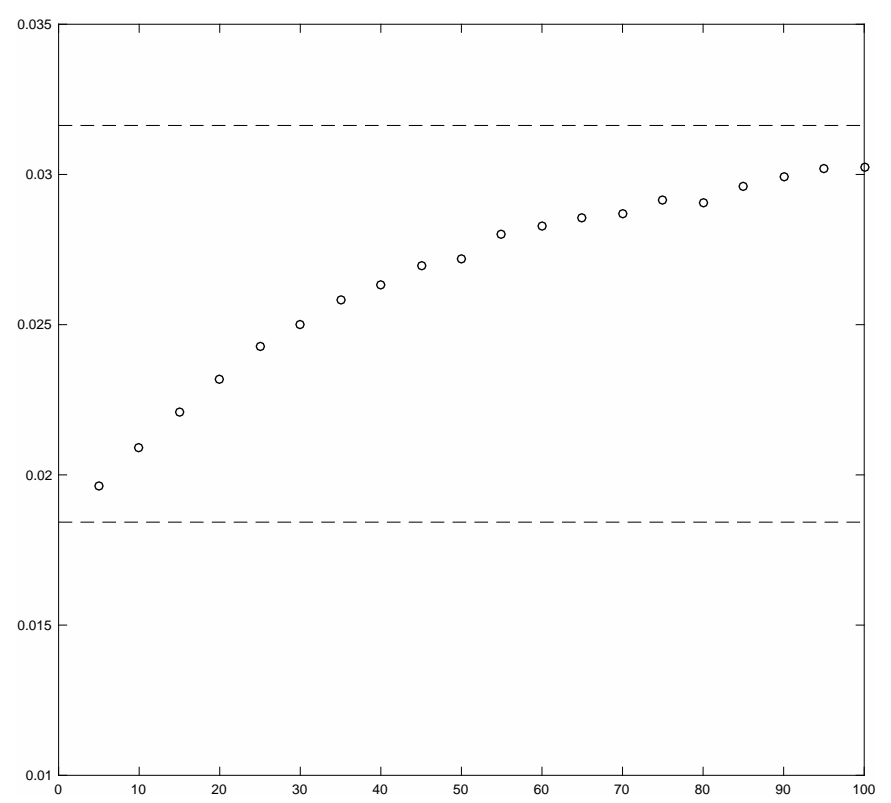

Figure 8: Asymptotic speed $c_{\infty}$ of the solutions of Eqs. (51) after one month as a function of the switch time $\tau_{h n}$. Parameter values are $\tau_{n}=24 \mathrm{~h}, \tau_{h}=48 \mathrm{~h}, D_{n}=6.6 \times 10^{-12} \mathrm{~cm}^{2} \mathrm{~s}^{-1}$, $D_{h}=6.6 \times 10^{-11} \mathrm{~cm}^{2} \mathrm{~s}^{-1}$. Shown are results for symmetric initial data given by Eq. (53) (o) and asymmetric initial data given by Eq. $(54)(\times)$. The dashed lines represent the asymptotic speeds of purely hypoxic (upper line) or normoxic (lower line) initial data in three dimensions in the absence of phenotype conversion (see text).

Thus, in this section we will consider the extension of Eqs.(1) to threedimensional scenarios, i.e. the equations

$$
\begin{aligned}
\frac{\partial u_{n}}{\partial t} & =D_{n} \triangle u_{n}+\frac{1}{\tau_{n}}\left(1-u_{n}-u_{h}\right) u_{n}+\frac{1}{\tau_{h n}} u_{h}, \\
\frac{\partial u_{h}}{\partial t} & =D_{h} \Delta u_{h}+\frac{1}{\tau_{h}}\left(1-u_{n}-u_{h}\right) u_{h}-\frac{1}{\tau_{h n}} u_{h} .
\end{aligned}
$$

To test the validity of our ideas we have repeated the most relevant numerical simulations of Sec. 3, but now for the fully 3D model given by Eqs. (51). Simulations were performed on $200 \times 200 \times 200$ grids using a second-order finite difference scheme (both in space and time). Computing time in a MacPro 12 core- workstation with $32 \mathrm{~Gb}$ of memory was around 4 hours/simulated month.

As an example, in Fig. 8 we show the dependence of the asymptotic speed after localized hypoxic events as a function of the switch time $\tau_{h n}$. The asymptotic velocity was computed by first finding the number of voxels in 3D with density $u_{n}(x, y, z, t)+u_{h}(x, y, z, t)$ larger than a threshold value $u_{t h}$. This provided a 
discretized volume $V(t)$. Then, the equation

$$
R_{s}(t)=\left(\frac{3 V(t)}{4 \pi}\right)^{1 / 3}
$$

was used to obtain an equivalent 'spherical radius' $R_{s}(t)$ at a given time. This number provides a measure of the tumor size (that is exact for fully spherical tumors). Finally the radial tumor growth speed was computed as $c_{s}(t)=R_{s}^{\prime}(t)$. The asymptotic radial growth speed, was computed as $c_{\infty}=\lim _{t \rightarrow \infty} c_{s}(t)$. In practice, with the family of initial data used, the asymptotic behavior was obtained for $t \sim 10$ days. We took the averaged value $\bar{c}=\int_{t_{1}}^{t_{2}} c_{s}(t) d t$ as an estimate for $c_{\infty}$, with $t_{1}=20$ days and $t_{2}=30$ days. This calculation was repeated for a broad range of values of the parameter $\tau_{h n}$ ranging between 0 and 100 hours.

Different types of initial data were tested. Specifically, symmetric initial data given by

$$
\begin{aligned}
& u_{h}(x, y, z, 0)=\left[1-200\left(x^{2}+y^{2}+z^{2}\right)\right]_{+}, \\
& u_{n}(x, y, z, 0)=0,
\end{aligned}
$$

and asymmetric initial data of the form

$$
\begin{aligned}
u_{h}(x, y, z, 0)= & 0.8 \operatorname{sech}\left(15 \sqrt{x^{2}+y^{2}+z^{2}}\right) \\
& +0.64 \operatorname{sech}\left(15 \sqrt{(x-0.12)^{2}+(y-0.04)^{2}+(z+0.17)^{2}}\right) \\
& +0.71 \operatorname{sech}\left(15 \sqrt{(x+0.08)^{2}+(y-0.12)^{2}+(z-0.05)^{2}}\right) \\
v_{h}(x, y, z, 0)= & 0 .
\end{aligned}
$$

The results are shown in Fig. 8. For comparison we computed the speed of propagation of: (i) purely normoxic data (lower dashed line in Fig. 8) and (ii) purely hypoxic data without phenotype conversion (i.e. Eq. (51b) without the last term) shown as the upper dashed line in Fig. 8. As in the one-dimensional case, the presence of the hypoxic component, even when transient, led to a significant speed boost of the normoxic component what confirms the main result of the previous analysis.

The calculations were repeated for different threshold values $0<u_{t h}<1$ and parameter choices. In all cases, the results found were independent of $u_{t h}$ and consistent with Fig. 8 and our previous 1D calculations detailed in Sec. 3.

\section{Therapeutical implications and conclusions}

Hypoxia is a characteristic feature of high-grade gliomas HGGs and arises first as a result of the proliferative activity of cells overcoming the capabilities of oxygen supply by the vasculature. In the case of gliomas there is an additional effect due to the secretion of prothrombotic factors that result in vessel failure. 
It is interesting to note that hypoxia is only marginal in low grade gliomas [55], where the vasculature remains mainly intact. Our results indicate that hypoxic events will result in an accelerated progression even when those hypoxic events are local in time, leading to a (may be diffuse and/or small in amplitude) front of invasive cells displaying the hypoxic phenotype (see e.g. Figure 3C of Zagzag et al [55]). This fact limits the potential efficacy of therapies targeting oxygenation alone, such as those described by Hatzikirou et al [25] or Martínez-González et al. [37] since it is not possible to stop completely the occurrence of hypoxic events in such an aggresive type of tumor.

Is there then an alternative to use re-oxygenation to favor the more proliferative yet less invasive phenotypes? The only possibility in the framework of the simple description used in this paper is to act on the switching time $\tau_{h n}$. In real situations $\tau_{h n}$ is not constant but typically increases with the number of hypoxia cycles experienced by cells until they reach to a state of physical balance with HIF- $1 \alpha$. This sequence of oxygen deprivation episodes drives genetic alterations in tumor cells so that HIF-1 $\alpha$ is accumulated in their nucleus even in oxic conditions and thus cells can not return to their previous state [47]. Therefore, $\tau_{h n}$ becomes larger than the typical proliferation time. Once the cells take so much time to revert to their less motile state in oxic conditions, the speed of the front increases in a sustained way (see Fig. 6). In fact, in vivo analysis of HIF-1 stabilization in well oxygenated tumor areas [55] supports long normalization times. However, if $\tau_{h n}$ could be kept small, as it happens in the normal cellular physiological state, the tumor invasion speed would drastically decrease to $c_{n}^{*}$ in oxic conditions. Thus, in order to be effective, a therapy involving enhanced oxygenation (or vascular normalization) should also act on HIF-1 $\alpha$ equilibrium simultaneously. This fact, in addition to other reasons, may be a reason for the failure of antiangiogenic therapies that lead to a radiographic response and increase in the progression free survival [20] but not to a real increase in survival of GBM patients [10]. Moreover it is known that in recurrent tumors the effect of antiangiogenic therapy is greatly reduced and a hypothesis for the lack of response after antiangiogenic treatment is an alteration of the tumor phenotype in a highly infiltrative compartment that is angiogenic-independent [5].

A final implication of our results affects the transition from low grade glioma (LGG) to higher grades for the GBMs. Although many factors may induce the transition, the simplest explanation involves the development of hypoxic areas [50]. This idea matches well with the fact that several distinctive features between LGGs and HGGs are related to the presence of hypoxia in the later ones: pseudopalisades and necrosis, microvascular proliferation and a higher cellularity originating the hypoxic events $[7,37]$. Our results show that preventing vessel failure and the cascade of malignant transformations associated to hypoxia may result in a delay in the appearance of the more invasive phenotype. Thus LGGs suspicious to undergo the malignant transformation might benefit from antithrombotic medication to avoid the degeneration into HGGs.

Because of the relevant role of hypoxia-inducible factors on the aggressiveness and tumorgenic capacity of glioma cells [34] stabilization of HIF- $1 \alpha$ has been also recently proposed as an attractive therapeutic target [47]. Our study points out 
yet another reason based on purely dynamical considerations. The stabilization of HIF-1 $\alpha$ would lead to smaller invasion speeds and thus to slower glioma progression if combined with therapies improving tumor oxygenation.

In summary, we have studied a simple model that shows the large impact of localized in time hypoxic events in the generation of waves of hypoxic cells that even when decaying on time may have a very relevant impact on the progression of gliomas. The mechanism is based on a low amplitude wave ("ghost" wave) of hypoxic cells that seeds the normal tissue in advance and accelerates the progression of the wave of more proliferative cells. The key parameter in this process is the time

We have also provided some theoretical results including necessary conditions for having persistent finite amplitude solitons composed of cells with an hypoxic phenotype. From a quantitative point of view, the hypoxic front wave has two 'components' one larger than the other. We have proven that the speed wave of the 'smallest' component of the hypoxic front wave converges to $c_{h n}^{*}$. The stability of those wave packets is a difficult mathematical problem, due to the essential spectrum and will be studied in the future.

\section{Appendix A. Critical points}

Let $\left(v_{n}, v_{h}\right)$ be a solution of (12) and define $V(\xi)=\left(V_{1}, V_{2}, V_{3}, V_{4}\right)=$ $\left(v_{n}, v_{n}^{\prime}, v_{h}, v_{h}^{\prime}\right)$, then

$$
\frac{d V}{d \xi}=F(V)
$$

where

$$
F(V):=\left(\begin{array}{cc}
V_{2} \\
-\frac{c}{D_{n}} V_{2}-\frac{1}{D_{n} \tau_{n}}\left(1-V_{1}-V_{3}\right) V_{1}-\frac{1}{D_{n} \tau_{h n}} V_{3} \\
-\frac{c}{D_{h}} V_{4}-\frac{1}{D_{h} \tau_{h}}\left(1-V_{1}-V_{3}\right) V_{3}+\frac{1}{D_{h} \tau_{h n}} V_{3}
\end{array}\right) .
$$

A point $\left(\bar{V}_{1}, \bar{V}_{2}, \bar{V}_{3}, \bar{V}_{4}\right)$ is an equilibrium of (A.1) if $F\left(\bar{V}_{1}, \bar{V}_{2}, \bar{V}_{3}, \bar{V}_{4}\right)=$ $(0,0,0,0)$. We will classify these linear equilibria by their generalized eigenspaces, according to the sign of the real part of the eigenvalues of the linearization, giving the decomposition $\mathbb{R}^{4}=E^{u} \oplus E^{s} \oplus E^{c}$ into the direct sum of unstable, stable and center eigenspaces.

Lemma 15. The points

$$
\left(\begin{array}{l}
0 \\
0 \\
0 \\
0
\end{array}\right), \quad\left(\begin{array}{l}
1 \\
0 \\
0 \\
0
\end{array}\right), \quad \frac{1-\tau_{h} / \tau_{h n}}{1 \tau_{n}-1 / \tau_{h}}\left(\begin{array}{c}
-1 / \tau_{h} \\
0 \\
1 / \tau_{n} \\
0
\end{array}\right)
$$

$\forall \tau_{n}, \tau_{h}, \tau_{h n} \in \mathbb{R}$ with $\tau_{n} \neq \tau_{h}$, are equilibrium points. 
Remark 16. For any $\tau_{n}, \tau_{h}, \tau_{h n} \in \mathbb{R} \backslash\{0\}$, the equilibrium $\left(\widetilde{V}_{1}, \widetilde{V}_{2}, \widetilde{V}_{3}, \widetilde{V}_{4}\right)^{T} \notin$ $\left(\overline{\mathbb{R}_{+}}\right)^{4}$

Let us denote by $A, \widehat{A}, \widetilde{A}$ the Jacobian matrices at the equilibria, i.e. $A:=$ $D F(0,0,0,0), \widehat{A}:=D F(1,0,0,0), \widetilde{A}:=D F\left(\widetilde{V}_{1}, \widetilde{V}_{2}, \widetilde{V}_{3}, \widetilde{V}_{4}\right)$. Let us denote their corresponding eigenvalues by $\sigma(A):=\left\{\lambda_{1+}, \lambda_{1-}, \lambda_{2+}, \lambda_{2-}\right\}, \sigma(\widehat{A}):=$ $\left\{\widehat{\lambda}_{1+}, \widehat{\lambda}_{1-}, \widehat{\lambda}_{2+}, \widehat{\lambda}_{2-}\right\}, \sigma(\widetilde{A})=\left\{\widetilde{\lambda}_{1+}, \widetilde{\lambda}_{1-}, \widetilde{\lambda}_{2+}, \widetilde{\lambda}_{2-}\right\}$

Theorem 17. The point $\left(\tau_{h} ;\left(V_{1}, V_{2}, V_{3}, V_{4}\right)\right)=\left(\tau_{h n} ;(0,0,0,0)\right)$ is a bifurcation point.

More precisely:

(a) Assume that $c>c_{n}^{*}$.

(a.i) If moreover $\tau_{h n}>\tau_{h}$, then $(0,0,0,0)$ is a stable equilibrium. Moreover

(a.i.1) If $c \geq c_{h n}^{*}$, then $(0,0,0,0)$ is a stable node.

(a.i.2) If $c<c_{h n}^{*}$, then $(0,0,0,0)$ is a stable node-focus.

(a.ii) If $\tau_{h n}<\tau_{h}$, then $(0,0,0,0)$ is a saddle point for any $c \in \mathbb{R}$.

(b) Assume that $c<c_{n}^{*}$.

(b.i) If moreover $\tau_{h n}>\tau_{h}$, then $(0,0,0,0)$ is an stable equilibrium.

(b.i.1) if $c \geq c_{h n}^{*}$, then $(0,0,0,0)$ is a stable focus-node.

(b.i.2) If $c<c_{h n}^{*}$, then $(0,0,0,0)$ is a stable focus.

(b.ii) If $\tau_{h n}<\tau_{h}$, then $(0,0,0,0)$ is a saddle point for any $c \in \mathbb{R}$.

Proof. We have to analyze the sign of the real part of the eigenvalues of $A$. Linearizing $f$ and computing for $(0,0,0,0)$ we get

$$
A:=D F(0,0,0,0)=\left(\begin{array}{cccc}
0 & 1 & 0 & 0 \\
-\frac{1}{D_{n} \tau_{n}} & -\frac{c}{D_{n}} & -\frac{1}{D_{n} \tau_{h n}} & 0 \\
0 & 0 & 0 & 1 \\
0 & 0 & \frac{1}{D_{h}}\left(\frac{1}{\tau_{h n}}-\frac{1}{\tau_{h}}\right) & -\frac{c}{D_{h}}
\end{array}\right)
$$

its spectrum being given by

$P_{0}(\lambda)=\operatorname{det}(A-\lambda I)=\frac{1}{D_{n} D_{h}}\left(D_{n} \lambda^{2}+c \lambda+\frac{1}{\tau_{n}}\right)\left(D_{h} \lambda^{2}+c \lambda+\frac{1}{\tau_{h}}-\frac{1}{\tau_{h n}}\right)$.

The eigenvalues can be written in the following way

$$
\lambda_{1 \pm}:=\frac{-c \pm \sqrt{c^{2}-4 D_{n} / \tau_{n}}}{2 D_{n}}, \quad \lambda_{2 \pm}:=\frac{-c \pm \sqrt{c^{2}-4 D_{h}\left(1 / \tau_{h}-1 / \tau_{h n}\right)}}{2 D_{h}} .
$$

Let us now analyze the sign of the real part of these eigenvalues 
(a) Obviously, if $c>c_{n}^{*}$ then the eigenvalues $\lambda_{1 \pm}$ are negative real numbers.

(a.i) If moreover $\tau_{h n}>\tau_{h}$, then $c_{h n}^{*} \in \mathbb{R}$.

(a.i.1) On the other side, if $c \geq c_{h n}^{*}$, then the eigenvalues $\lambda_{2 \pm}$ are negative real numbers and as a consequence, all the eigenvalues of the Jacobian matrix are negative real numbers and the trivial equilibria is a estable node.

(a.i.2) If $c<c_{h n}^{*}$, then $\lambda_{2 \pm} \in \mathbb{C}^{-}$. As a conclusion, $\sigma(A) \subset \mathbb{C}^{-}$, has two real eigenvalues and two complex eigenvalues, and $(0,0,0,0)$ is a stable node-focus.

(a.ii) If $\tau_{h n}<\tau_{h}$, then $\lambda_{2-}<0<\lambda_{2+}$. Therefore, $\sigma(A) \subset \mathbb{R}$, and has positive eigenvalue concluding that $(0,0,0,0)$ is a saddle point for any $c \in \mathbb{R}$.

(b) If $c<c_{n}^{*}$, then $\lambda_{1 \pm} \in \mathbb{C}^{-}$.

(b.i) If moreover $\tau_{h n}>\tau_{h}$, then $c_{h n}^{*} \in \mathbb{R}$.

(b.i.1) if $c \geq c_{h n}^{*}$, then $\sigma(A) \subset \mathbb{C}^{-}$, and $(0,0,0,0)$ is a stable focus-node.

(b.i.2) If $c<c_{h n}^{*}$, then $\lambda_{2 \pm} \in \mathbb{C}^{-}, \sigma(A) \subset \mathbb{C}^{-}$, and $(0,0,0,0)$ is a stable focus.

(b.ii) If $\tau_{h n}<\tau_{h}$, then $\lambda_{2-}<0<\lambda_{2+}$, and $(0,0,0,0)$ is a saddle point for any $c \in \mathbb{R}$.

Remark 18. Let us remark that if $c=c_{n}^{*}$, then $\lambda_{1+}=0$. Also if $\tau_{h n}=\tau_{h}$, then, by definition, $c_{h n}^{*}=0$, and $\lambda_{2+}=0$.

Theorem 19. For any $0<\tau_{n}<\infty$ and $\tau_{h n}>0$, the equilibrium $\left(V_{1}, V_{2}, V_{3}, V_{4}\right)=$ $(1,0,0,0)$ is a saddle point, $\operatorname{dim}\left(E^{u}\right)=\operatorname{dim}\left(E^{s}\right)=2$, and $E^{u}$ is tangent to the space $\operatorname{span}\left[\left(1, \widehat{\lambda}_{1+}, 0,0\right),\left(0,0,1, \widehat{\lambda}_{2+}\right)\right]$ at $(1,0,0,0)$.

Proof. Since

$$
\widehat{A}=D F(1,0,0,0)=\left(\begin{array}{cccc}
0 & 1 & 0 & 0 \\
\frac{1}{D_{n} \tau_{n}} & -\frac{c}{D_{n}} & \frac{1}{D_{n}}\left(\frac{1}{\tau_{n}}-\frac{1}{\tau_{h n}}\right) & 0 \\
0 & 0 & 0 & 1 \\
0 & 0 & \frac{1}{D_{h} \tau_{h n}} & -\frac{c}{D_{h}}
\end{array}\right),
$$

its spectrum is given by

$$
P_{1}(\lambda)=\frac{1}{D_{n} D_{h}}\left(D_{n} \lambda^{2}+c \lambda-\frac{1}{\tau_{n}}\right)\left(D_{h} \lambda^{2}+c \lambda-\frac{1}{\tau_{h n}}\right) .
$$


The eigenvalues are given by

$$
\widehat{\lambda}_{1 \pm}:=\frac{-c \pm \sqrt{c^{2}+4 D_{n} / \tau_{n}}}{2 D_{n}}, \quad \widehat{\lambda}_{2 \pm}:=\frac{-c \pm \sqrt{c^{2}+4 D_{h} / \tau_{h n}}}{2 D_{h}} .
$$

Whenever $\tau_{h n}, \tau_{n}>0$, the eigenvalues $\widehat{\lambda}_{1 \pm}, \widehat{\lambda}_{2 \pm} \in \mathbb{R}$ and satisfy $\widehat{\lambda}_{1-}<0<$ $\widehat{\lambda}_{1+}, \quad \hat{\lambda}_{2-}<0<\widehat{\lambda}_{2+}$, and as a consequence, the non-trivial equilibria $(1,0,0,0)^{T}$ is a saddle point, and $\operatorname{dim}\left(E^{u}\right)=\operatorname{dim}\left(E^{s}\right)=2$. Moreover, it is not difficult to prove that $E^{u}$ is tangent to $\operatorname{span}\left[\left(1, \widehat{\lambda}_{1+}, 0,0\right),\left(0,0,1, \widehat{\lambda}_{2+}\right)\right]$ at $(1,0,0,0)$, which completes the proof.

\section{Acknowledgements}

This work has been partially supported by the Ministerio de Economía y Competitividad (Spain), under grants MTM2012-31073, MTM2015-71200-R and MTM2012-31298 and by Consejería de Educación y Ciencia de la Junta de Comunidades de Castilla-La Mancha under grant PEII-2014-031-P. 
[1] Anderson A (2005) A hybrid mathematical model of solid tumour invasion: the importance of cell adhesion. Math Med Biol. 22:163-186

[2] Arendt W, Batty CJK (1993) Exponential stability of a diffusion equation with absorption, Differential Integral Equations 6,5: 1009-1024.

[3] Ayuso JM, Monge R, Martínez-González A, Llamazares GA, Berganzo J, Hernández-Laín A, Santolaria J, Doblaré M, Sánchez-Gómez P, PérezGarcía VM, Ochoa I, Fernández LJ (2016) Glioblastoma on a microfluidic chip: Generating pseudopalisades and enhancing cancer cellś aggressiveness through thrombotic events, Neuro-oncology

[4] Bashkara VK, Mohanam I, Rao JS, Mohanam S (2012) Intermittent Hypoxia Regulates Stem-like Characteristics and Differentiation of Neuroblastoma Cells. PLoS ONE 7:e30905

[5] Beal K, Abrey LE, Gutin PH (2011) Antiangiogenic agents in the treatment of recurrent or newly diagnosed glioblastoma: analysis of single-agent and combined modality approaches. Radiat Oncol 6:2.

[6] Berens ME, Giese A (1999) “...those left behind." Biology and oncology of invasive glioma cells. Neoplasia 1:208-219.

[7] Brat DJ, Van Meir EG (2004). Vaso-occlusive and prothrombotic mechanims associated with tumor hypoxia, necrosis, and accelerated growth in glioblastoma. Lab Invest 84:397-405.

[8] Brazhnik PK and Tyson JJ (2000) On Traveling Wave Solutions of Fisher's Equation in Two Spatial Dimensions, SIAM Journal on Applied Mathematics, 60:371- 391

[9] Brézis H (2011)Functional analysis, Sobolev spaces and partial differential equations. Universitext. Springer, New York. ISBN 978-0-387-70913-0.

[10] Butowski N (2011) Anti-angiogenic therapy in glioma. Clin Translat Oncol 13:294-300

[11] Chi JT, Wang Z, Nuyten DSA, Rodriguez EH, Schaner ME, Salim A, Wang Y, Kristensen GB, Helland A, Borresen-Dale AL, Giaccia A, Longaker MT, Hastie T, Yang GP, van de Vijver MJ, Brown PO (2006) Gene Expression Programs in Response to Hypoxia: Cell Type Specificity and Prognostic Significance in Human Cancers. PLoS Medicine 3:e47

[12] Das B, Tsuchida R, Malkin D, Koren G, Baruchel S, et al. (2008) Hypoxia enhances tumor stemness by increasing the invasive and tumorigenic side population fraction. Stem Cells 26:1818-1830.

[13] Dutzmann S, Gessler F, Harter PN, Gerlach R, Mittelbronn M, Seifert V, Kogel D (2010) The pro-migratory and pro-invasive role of the procoagulant tissue factor in malignant gliomas. Cell Adhesion \& Migration 4:515-522. 
[14] Coddington EA and Levinson N (1955) Theory of ordinary differential equations. McGraw-Hill Book Company, Inc., New York-Toronto-London.

[15] Ebos JML, Kerbel RS. (2011). Antiangiogenic therapy, impact on invasion, disease progression, and metastasis. Nat. Rev. Clin. Oncol. 8:210-221.

[16] Elstner A, Holtkamp N, von Deimling A (2007) Involvement of Hif-1 in desferrioxamine-induced invasion of glioblastoma cells, Clin Exp Metastasis 24:57-66.

[17] Evans SM, Judy KD, Dunphy I, Jenkins WT, Hwang WT, Nelson PT, Lustig RA, Jenkins K, Magarelli DP, Hahn SM, Collins RA, Grady S, Koch CJ (2004) Hypoxia Is Important in the Biology and Aggression of Human Glial Brain Tumors. Clin Cancer Res 10:8177-8184.

[18] Fedotov S, Iomin A, Ryashko L (2011) Non-Markovian models for migration-proliferation dichotomy of cancer cells: Anomalous switching and spreading rate. Phys Rev E 84:061131.

[19] Fisher RA (1937) The Wave of Advance of Advantageous Genes. Ann. Eugen.,7, 335-369.

[20] Friedman HS, Prados M, Wen PY, Mikkelsen T, Schiff D, Abrey LE, Yung WK, Paleologos N, Nicholas MK, Jensen R, Vredenburgh J, Huang J, Zheng M, Cloughesy T (2009) Bevacizumab alone and in combination with irinotecan in recurrent glioblastoma. J Clin Oncol 27:4733-4740.

[21] Giese, A., Loo, M. A., Tran, N., Haskett, D., Coons, S. W. and Berens, M. E., 1996. Dichotomy of astrocytoma migration and proliferation. Int. J. Cancer, 67: 275-282.

[22] Giese A, Bjerkvig R, Berens ME, Westphal M (2003). Cost of migration: invasion of malignant gliomas and implications for treatment. J. Clin. Oncol. 21:1624-1636.

[23] Hale, Jack K. and Koçak, Hüseyin (1991) Dynamics and bifurcations, Texts in Applied Mathematics, Vol. 3, Springer-Verlag, New York.

[24] Hanahan D, Weinberg RA (2011) The hallmarks of cancer: The next generation. Cell 144:646-674

[25] Hatzikirou H, Basanta D, Simon M, Schaller K, Deutsch A (2012) 'Go or Grow': the key to the emergence of invasion in tumour progression? Math Med Biol 29: 49-65

[26] Henry D (1981) Geometric theory of semilinear parabolic equations. Lecture Notes in Mathematics. Springer-Verlag, Berlin 840.

[27] Iomin A (2006) Toy model of fractional transport of cancer cells due to self-entrapping. Phys Rev E 73:061918 
[28] Jensen RL (2009) Brain tumor hypoxia: tumorigenesis, angiogenesis, imaging, pseudoprogression, and as a therapeutic target. J. Neurooncol. 92:317335 .

[29] Jewell UR, Kvietikova I, Scheid A, Bauer C, Wenger RH, Gassmann M (2001) Induction of HIF- $1 \alpha$ in response to hypoxia is instantaneous, The FASEB J 15:1312

[30] Ke LD, Shi YX, Im SA, Chen X, Yung WK., 2000, The relevance of cell proliferation, vascular endothelial growth factor, and basic fibroblast growth factor production to angiogenesis and tumorigenicity in human glioma cell lines, Clin Cancer Res 6:2562-2572.

[31] Kirkby N, Jefferies SJ, Jena R, Burnet NG (2007) A mathematical model of the treatment and survival of patients with high-grade brain tumours, J. Theor. Biol. 245:112-124.

[32] Kolmogorov AN, Petrovskii IG, Piskunov NS 1937. A study of the equation of diffusion with increase in the quantity of matter, and its application to a biological problem. Bjul. Moskovskovo Gos. Univ. 17, 1-72.

[33] Koppenol WH, Bounds PL, Dang CV (2011) Otto Warburg's contribution to current concepts of cancer metabolism. Nature Rev Cancer 11:325-337

[34] Li Z, Bao S, Wu Q, Wang H, Eyler C, Sathornsumetee S, Shi Q, Cao Y, Lathia J, McLendon RE, Hjelmeland AB, Rich JN (2009) Hypoxiainducible factors regulate tumorigenic capacity of glioma stem cells. Cancer Cell 15:501-513.

[35] Majmundar AJ, Wong WJ, Simon MC, Hypoxia-Inducible Factors and the Response to Hypoxic Stress. Molecular Cell 40:294-309

[36] Mangional A, Anile C, Pompucci A, Capone G, Rigante L, De Bonis P (2010) Glioblastoma therapy: Going beyond Hercules Columns. Expert Rev Neurother 10:507-514.

[37] Martínez-González A, Calvo GF, Pérez-Romansanta LA, Pérez-García VM (2012) Hypoxic Cell Waves around Necrotic Cores in Glioblastoma: A Biomathematical Model and its Therapeutic Implications. Bull Math Biol. 74(12): 2875-2896.

[38] Martínez-González A, Durán-Prado M, Calvo GF, Alcaín FJ, PérezRomansanta LA, Pérez-García VM (2015) Combined therapies of antithrombotics and antioxidants delay in silico brain tumor progression. Math Med \& Biol. 32: 239-262

[39] Mendoza-Juez B, Martínez-González A, Calvo GF, Pérez-García VM (2011) A mathematical model for the glucose-lactate metabolism of in vitro cancer cells. Bull Math Biol 74:1125-1142 
[40] Onishi M, Ichikawa T, Kurozumi K, Date l (2011). Angiogenesis and invasion in glioma. Brain Tumor Pathol. 28:13-24.

[41] Pérez-García VM, Bogdanska M, Martínez-González A, Belmonte-Beitia J, Schucht P, Pérez-Romasanta LA (2015) Delay effects in the response of lowgrade gliomas to radiotherapy: a mathematical model and its therapeutical implications. Math Med Biol. 32:307-329.

[42] Pérez-García VM, Pérez-Romasanta LA (2016) Extreme protraction for low-grade gliomas: theoretical proof of concept of a novel therapeutical strategy. Math Med Biol. doi:10.1093/imammb/dqv017 (in press).

[43] Pham K, Chauviere A, Hatzikirou H, Li X, Byrne HM, Cristini V, Lowengrub J (2011) Density-dependent quiescence in glioma invasion: instability in a simple reactio-diffusion model for the migration/proliferation dichotomy. Jour Biol Dyn 6:54-71

[44] Ramis-Conde I, Chaplain M, Anderson A (2008) Mathematical modelling of cancer cell invasion of tissue. Math Comput Modell. 47:533-545.

[45] Ranalli NJ, Evans SM, Judy KD (2009) Hypoxia in brain tumors: A review of the literature. Neurosurg Quart 19:1-12

[46] Rong Y, Durden DL, Van Meir EG \& Brat DJ (2006). 'Pseudopalisading' necrosis in glioblastoma: a familiar morphologic feature that links vascular pathology, hypoxia, and angiogenesis. J Neuropathol Exp Neurol 65:529-539.

[47] Semenza GL (2003) Targeting HIF-1 for cancer therapy. Nat Rev Cancer 3:721-732.

[48] Smoller J (1994) Shock waves and reaction-diffusion equations. Fundamental Principles of Mathematical Sciences, V. 258, $2^{\text {nd }}$ edition, Springer-Verlag, New York, ISBN 0-387-94259-9.

[49] Stein AM, Demuth T, Mobley D, Berens M, Sander LM (2007) A Mathematical Model of Glioblastoma Tumor Spheroid Invasion in a ThreeDimensional In Vitro Experiment. Biophys J 92:356-365

[50] Swanson KR, Rockne RC, Claridge J, Chaplain MA, Alvord EC Jr, Anderson AR (2011) Quantifying the role of angiogenesis in malignant progression of gliomas: In silico modeling integrates imaging and histology. Cancer Research 71:7366

[51] Tektonidis M, Hatzikirou H, Chauviere A, Simon M, Schaller K, Deutsch A (2011) Identification of intrinsic in vitro cellular mechanisms for glioma invasion. J Theor Biol 287:131-147

[52] Toffoli S, Michielis C (2008) Intermittent hypoxia is a key regulator of cancer cell and endothelial cell interplay in tumours. FEBS Journal 275:29913002 
[53] Wang CH, Rockhill JK, Mrugala M, Peacock DL, Lai A, Jusenius K, Wardlaw JM, Cloughesy T, Spence AM, Rockne R, Alvord EC, Jr \& Swanson KR (2009). Prognostic significance of growth kinetics in newly diagnosed glioblastomas revealed by combining serial imaging with a novel biomathematical model. Cancer Res. 69:9133-9140.

[54] Wen PY, Kesari S (2008). Malignant gliomas in adults. N Engl J Med 359:492-507.

[55] Zagzag D, Zhong H, Scalzitti JM, Laughner E, Simons JW, Semenza GL (2000) Expression of hypoxia-inducible factor $1 \alpha$ in brain tumors: association with angiogenesis, invasion, and progression. Cancer 88:2606-2618. 